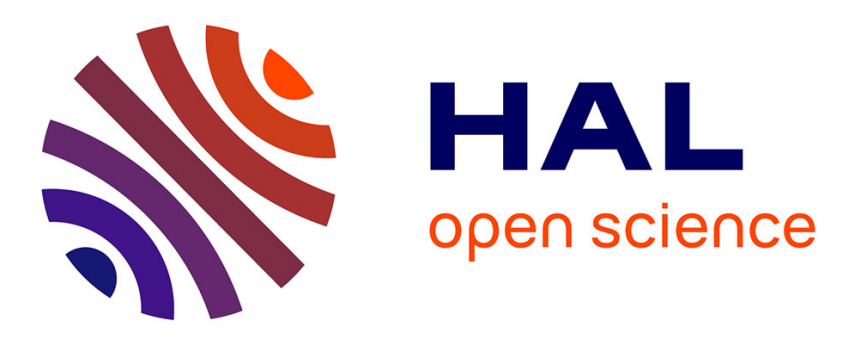

\title{
Oxygen Adsorption on Graphene-Encapsulated Palladium Nanoparticles Imaged by Kelvin Probe Force Microscopy
}

Henrik Grönbeck, Clemens Barth

\section{- To cite this version:}

Henrik Grönbeck, Clemens Barth. Oxygen Adsorption on Graphene-Encapsulated Palladium Nanoparticles Imaged by Kelvin Probe Force Microscopy. Journal of Physical Chemistry C, 2019, 123 (40), pp.24615-24625. 10.1021/acs.jpcc.9b07377 . hal-02346893

\section{HAL Id: hal-02346893 \\ https://hal.science/hal-02346893}

Submitted on 5 Nov 2019

HAL is a multi-disciplinary open access archive for the deposit and dissemination of scientific research documents, whether they are published or not. The documents may come from teaching and research institutions in France or abroad, or from public or private research centers.
L'archive ouverte pluridisciplinaire HAL, est destinée au dépôt et à la diffusion de documents scientifiques de niveau recherche, publiés ou non, émanant des établissements d'enseignement et de recherche français ou étrangers, des laboratoires publics ou privés. 


\title{
Oxygen Adsorption on Graphene Encapsulated Palladium Nanoparticles Imaged by Kelvin Probe Force Microscopy
}

\author{
Henrik Grönbeck ${ }^{\dagger}$ and Clemens Barth*,‡ \\ Chalmers University of Technology, Department of Physics and Competence Centre for \\ Catalysis, SE-41296 Göteborg, Sweden, and Aix-Marseille Université, CNRS, CINaM UMR \\ 7325, 13288 Marseille, France \\ E-mail: barth@cinam.univ-mrs.fr
}

${ }^{*}$ To whom correspondence should be addressed

${ }^{\dagger}$ Chalmers University of Technology, Department of Physics and Competence Centre for Catalysis, SE41296 Göteborg, Sweden

¥Aix-Marseille Université, CNRS, CINaM UMR 7325, 13288 Marseille, France 


\begin{abstract}
Graphene encapsulated metal nanoparticles (G@NPs) offer a possibility to observe confined reactions in the nanocontainer formed by the NP's facets and graphene. However, direct experimental detection of adsorbed atomic and molecular species under the graphene cover is still challenging and the mechanisms of intercalation and adsorption are not well understood. Here we show that Kelvin probe force microscopy (KPFM) can largely contribute to the understanding of adsorption and desorption at the single NP level, which we exemplify by comparing oxygen adsorption experiments obtained at asprepared PdNPs and G@PdNPs, both supported on highly oriented pyrolytic graphite (HOPG) and studied under ultra-high vacuum (UHV) conditions. We show that oxygen adsorption at room temperature occurs at a much higher partial oxygen pressure on G@PdNPs compared to as-prepared PdNPs. Similarly, the removal of oxygen via a reaction with the residual gas of the UHV is slower on the G@PdNPs compared to as-prepared PdNPs. The differences can be explained by a limited facility for reactant and product molecules to enter and desorb from the nanocontainer via the defects of the graphene. Observed WF changes are supported by assisting density functional theory (DFT) calculations.
\end{abstract}




\section{INTRODUCTION}

Graphene encapsulated nanoparticles (G@NPs) have lately come into focus of several disciplines in nanosciences. Apart from NPs made from $\mathrm{Pt}^{1-3}, \mathrm{Pd}^{4-6}, \mathrm{Au}^{7}, \mathrm{Fe}^{8}, \mathrm{Co}^{9,10}$ and $\mathrm{Cu}^{4,11}$ as well as $\mathrm{FeCo}^{12,13}$ and $\mathrm{NiCo}^{14,15}$, also semiconducting NPs made from silicon ${ }^{16,17}$ and even oxide NPs such as $\mathrm{Fe}_{2} \mathrm{O}_{3}{ }^{18}$ and $\mathrm{TiO}_{2}{ }^{19}$ have been explored. Core-shell G@NPs exhibit several advantages in comparison to their pure counterparts with potential applications in surface enhanced Raman scattering (SERS) ${ }^{20,21}$, lithium ion batteries (LiBs) ${ }^{16-18,22,23}$, nanobiotechnology ${ }^{24}$ and hydrogen storage solutions ${ }^{25}$. In heterogeneous catalysis, it has been suggested that a protecting graphene shell could prevent degradation and metal sintering ${ }^{10,14,26,27}$ and that the graphene-NP ensemble could alter the catalytic properties ${ }^{26}$. Furthermore, graphene protected NPs provide a system for catalysis under cover ${ }^{28,29}$ where chemical reactions take place in the nanocontainer formed by the graphene wall and NP facets. In this way, the G@NP systems offers a possibility to investigate the effects of confinement on catalytic reactions. As the reactants and products should enter and leave the container via defect sites, it can be anticipated that the reactions can become mass transfer limited.

To detect adsorption or desorption phenomenon at the single NP level, a direct measure is desired. In principle, scanning tunneling microscopy (STM) can detect atomic or molecular species under a graphene sheet as shown on single crystal surfaces ${ }^{30}$. However, the imaging is challenging and has never been done on nanometer large NPs, and STM is restricted to conducting surfaces only. A simple experimental method for detecting adsorption and desorption on metal surfaces is to measure related work function (WF) changes of the metal, as exemplified by the macroscopic Kelvin probe technique on single crystal surfaces. For instance, oxygen and hydrogen dissociatively adsorb on $\mathrm{Pd}(111)^{31-33}$ and increase the WF depending on the coverage: at room temperature, saturation values of $+0.8^{34}$ and $+0.3 \mathrm{eV}^{31}$ are found for oxygen and hydrogen, respectively. Carbon monoxide increases the WF by up to $+1.0 \mathrm{eV}^{35}$ whereas water decreases the $\mathrm{WF}$ of metals in general ${ }^{36}$, as in the case of palladium with saturation values of around $\sim-0.8 \mathrm{eV}^{37}$. A strong $\mathrm{WF}$ reduction is also 
observed when carbon is dissolved in a subsurface region $(\sim-1 \mathrm{eV})$, which we exemplified at PdNPs ${ }^{6}$. One possibility would therefore be to monitor changes of the NP's WF when G@NPs are exposed to an gaseous environment: if adsorption or desorption of atomic or molecular species takes place on the NP's facets, the WF of the G@NP should change proportional to the adsorbate concentration ${ }^{31,34}$. The WF of a single NP can be measured by Kelvin probe force microscopy $(\mathrm{KPFM})^{38}$, which is an implementation of Kelvin probe into noncontact atomic force microscopy (nc-AFM) ${ }^{38}$. Thanks to the spacial resolution at the nanometer scale and the $\mathrm{mV}$ resolution in WF, KPFM yields WF information of nanometer sized islands and $\mathrm{NPs}^{6,39-41}$.

In this work we show that KPFM does indeed contribute to the understanding of adsorption and desorption at the single NP level by measuring changes of the NP's WF. The technique helps to reveal adsorption phenomena of oxygen at room temperature (RT) and under ultra-high vacuum conditions (UHV) on as-prepared palladium NPs (PdNPs) and graphene encapsulated palladium NPs (G@PdNPs), which are supported on highly oriented pyrolytic graphite (HOPG). Palladium is an important NP material in catalysis and graphene can easily be grown on PdNPs ${ }^{5,6}$. The practical advantage of HOPG is that the very wide terraces are inert towards oxygen and other molecules at $\mathrm{RT}^{42}$, so that the HOPG surface always remains at its well-defined WF and therefore functions as a reference surface for KPFM.

\section{METHODS}

Synthesis of PdNPs on HOPG. Clean surfaces are prepared by cleaving HOPG in air and following annealing at $\sim 650^{\circ} \mathrm{C}$ in UHV during several hours. The PdNPs are grown by evaporating neutral palladium atoms onto the HOPG sample. During the growth, the HOPG substrate is held at a temperature between 450 and $500{ }^{\circ} \mathrm{C}$. For the synthesis of graphene on the PdNPs, the UHV chamber is back-filled with ethylene via a leakage valve 
at a pressure between 1.0 and $2.0 \times 10^{-6} \mathrm{mbar}$, and the dosage is determined by the exposure time. During the ethylene exposure, the sample is held at a constant temperature between 670 and $740{ }^{\circ} \mathrm{C}$. In oxygen adsorption experiments, the UHV chamber is back-filled with molecular oxygen via a leakage valve at a pressure between $3.0 \times 10^{-7}$ and $4.0 \times 10^{-5} \mathrm{mbar}$, whereas the dosage is also determined by the exposure time. Adsorption experiments are done at room temperature and during the SPM imaging (in operando experiments).

Scanning Probe Microscopy (SPM). STM, nc-AFM and KPFM experiments are performed in the same UHV chamber $\left(1 \times 10^{-10} \text { mbar base pressure }\right)^{76}$ with a room temperature AFM/STM. KPFM is used in the frequency modulation mode ${ }^{77}$ and applied during the topography nc-AFM imaging mode. During the scanning of the surface, the electrostatic tip-surface interaction is minimized at each image point by the bias voltage, yielding the contact potential difference $(\mathrm{CPD})$ between tip and surface defined as $\mathrm{CPD}=\left(\phi_{\text {sample }}-\phi_{\text {tip }}\right) / e$. A so-called work function image of the CPD is simultaneously obtained with the topography nc-AFM image. The contrast of a WF image is directly related to WF differences on the surface. A bright and in particular orange/yellow contrast in WF images corresponds to a high WF whereas a violet and black contrast corresponds to a low WF. Note that for a better data reading, the CPD values for HOPG are shifted onto zero such that the values of the profiles are directly related to the WF difference between the PdNPs and HOPG.

Theory. DFT is used as implemented in the $\mathrm{Dmol}^{3}$ program ${ }^{78,79}$. The calculations are performed either within the local density approximation $(\mathrm{LDA})^{80}$. LDA is considered as this functional thanks to error cancellation is known to provide good results for surface properties such as surface energies and work-functions ${ }^{81,82}$. As binding energies generally are strongly overestimated in LDA, we do not report any adsorption of diffusion energies. The $\operatorname{Pd}(111)$ surface is modeled with 7 layers using four different surface cells; $\sqrt{3} \times \sqrt{3}, 2 \sqrt{3} \times 2 \sqrt{3}$, $p(2 \times 2)$ and $p(5 \times 5)$. Geometry optimization is performed using the BFGS method. The work function $(\phi)$ is the energy needed to remove an electron from the bulk of palladium to the vacuum. It is calculated according to: $\phi=V_{\text {vacuum }}-\epsilon_{F}$, where $V_{\text {vacuum }}$ is the electrostatic 
potential in the vacuum region and $\epsilon_{F}$ is the Fermi energy.

More details about the materials, work function values and sample preparation as well as details about STM, nc-AFM, KPFM and DFT calculations can be found in the Supporting Information.

\section{RESULTS}

Oxygen Exposure on As-Prepared PdNPs. Figure 1a shows a topography (z) and WF image of PdNPs, after their high-temperature growth at $476{ }^{\circ} \mathrm{C}$ on $\mathrm{HOPG}$ in UHV. In the following, such NPs are referred to as as-prepared PdNPs. Owing to their 3D shape of a top-truncated tetrahedron, the NPs exhibit well-known shapes from triangles to hexagons via various truncated shapes, with the NPs' edges forming always angles of $60^{\circ}$ and $120^{\circ} 41$. The top facets are atomically flat and are in their (111) surface orientation, with the side facets having (111) and (001) orientations ${ }^{6}$. The NPs are attached at steps of the HOPG surface and have side lengths, which can vary between 10 and $50 \mathrm{~nm}$, whereas their heights generally can reach $10 \mathrm{~nm}$ and more.

A $+0.4 \pm 0.1 \mathrm{eV}$ small WF difference can be found between the PdNPs and HOPG in Figure 1a (see profile in Figure 1a for one NP), which is much smaller than the expected WF difference of $\sim+1.1 \mathrm{eV}$ between pristine Pd(111) and HOPG $\left(\phi_{\mathrm{HOPG}, \mathrm{lit}}=4.5 \pm 0.1 \mathrm{eV}\right.$, $\phi_{\mathrm{Pd}, \mathrm{lit}}=5.6 \pm 0.1 \mathrm{eV}$, see Table S1 and $\mathrm{S} 2$ in the Supporting Information). The reason for the small difference is that carbon is detached from the HOPG steps during the NP growth at $476^{\circ} \mathrm{C}$ and occupies subsurface sites in the NP, reducing the WF of the palladium $\mathrm{NP}^{6}$.

The image series in Figure $1 \mathrm{~b}$ and e shows the same NPs, successively recorded during an exposure of molecular oxygen at room temperature. For each image, the scanning is started at the bottom of the image and finishes at the top after about 35 minutes. Thus, the upward oriented slow scanning direction is a measure of the measurement time, which is represented by the blue and orange arrows in all images of Figure 1. Important to note is 

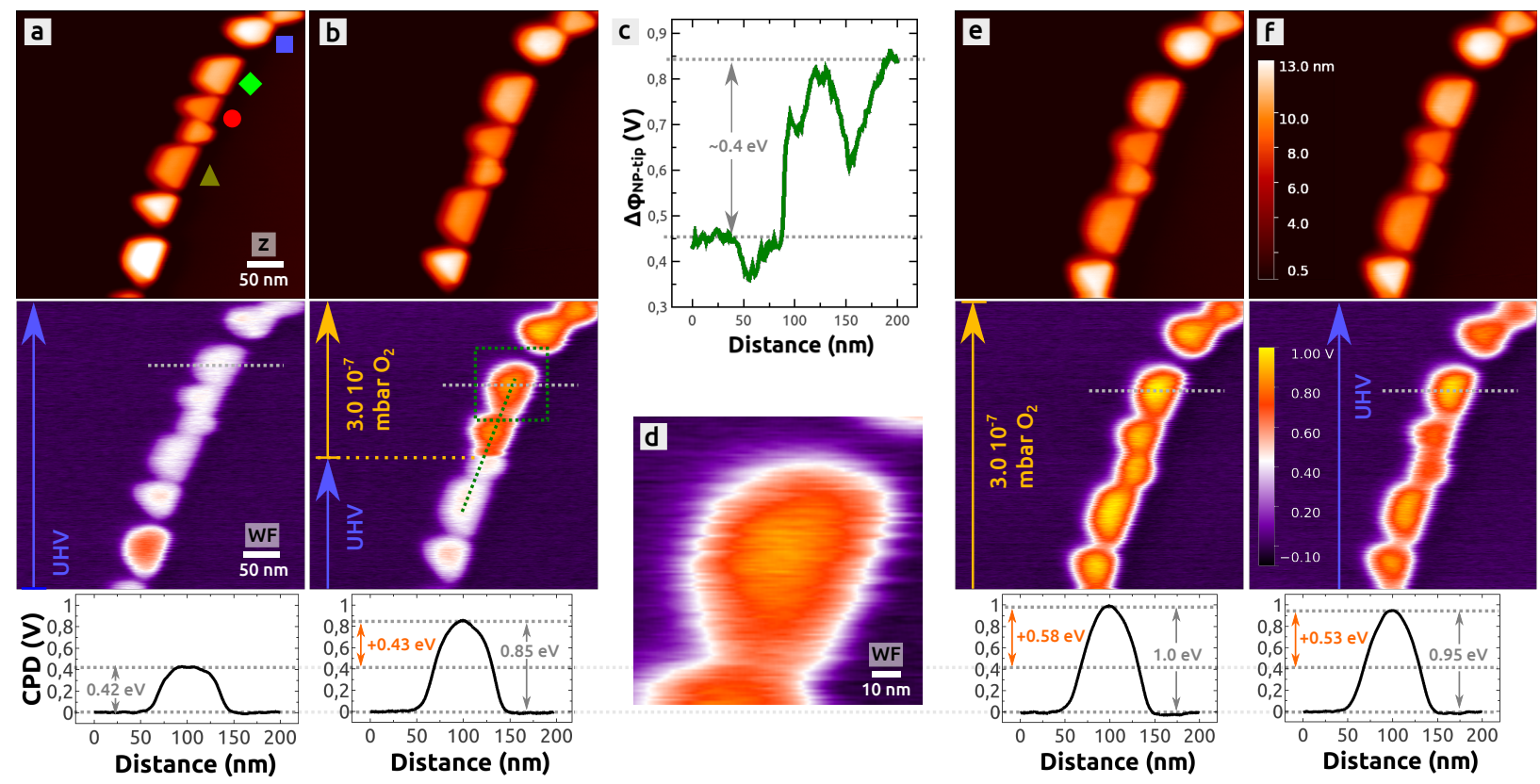

Figure 1: As-prepared PdNPs imaged by KPFM during an exposure of $\mathrm{O}_{2}$ at room temperature $\left(771 \mathrm{~L} @ p_{\mathrm{O} 2}=3 \times 10^{-7} \mathrm{mbar}, \mathrm{t}_{\text {exposure }}=57 \mathrm{~min}\right)$. One KPFM experiment is represented by a vertically arranged topography (top) and WF image (bottom), with a representative WF profile underneath taken from the gray dotted line in each WF image. The profiles show the contrast for one and the same NP. The WF images have all the same color scale (See Figure S1 in the Supporting Information). The orange arrows show the $\mathrm{O}_{2}$ exposure whereas blue arrows correspond to the imaging in UHV (no $\mathrm{O}_{2}$ ). (c) Profile taken from the WF image in (b) at the green dotted line. (d) Region of the dotted green square in the WF image in (b). $\mathrm{NP}$ growth: $2.0 \mathrm{ML}$ at $476{ }^{\circ} \mathrm{C}, \mathrm{KPFM}$ parameters: $\triangle f=-8.2 \mathrm{~Hz}, v=0.5 \mathrm{~Hz}$, $U_{\mathrm{ac}}=650 \mathrm{mV}$ and $f_{\mathrm{ac}}=634 \mathrm{~Hz}$.

that one scanning line needs about $t_{\text {line }}=4$ seconds (scanning speed $v=0.5 \mathrm{~Hz}$, scanning in forward and backward direction).

Up to the middle of image Figure 1b, the NPs are imaged in UHV (blue arrows) whereas starting from the middle, the UHV chamber is backfilled with molecular oxygen (orange arrows). A partial oxygen pressure of $p_{\mathrm{O} 2}=3.0 \times 10^{-7} \mathrm{mbar}$ is used so that after the scanning of one line, a dosage of $\sim 1$ Langmuir $(\mathrm{L})$ is achieved $\left(t_{\text {line }} \times p_{\mathrm{O} 2} / 1.33 \times 10^{-6} \mathrm{mbar} \approx 0.9 \mathrm{~L}\right)$. Within a few scanning lines, the WF of the PdNPs increases on average by $+0.4 \pm 0.1 \mathrm{eV}$, 
which can be seen by the large contrast change in the WF image (from white to orange in Figure 1b) and in the profile of the representative NP below the WF image. In Figure 1c, a profile along the row of NPs (green dotted line in Figure 1b) shows the immediate WF increase more clearly. After this strong increase of the WF, the WF contrast of the top facets is homogeneous (Figure 1d). The WF remains almost constant with only a slight increase for all NPs in the following KPFM measurement in Figure 1e: after about 30 minutes of oxygen dosage, the mean WF has increased onto $+0.6 \pm 0.1 \mathrm{eV}$. The last image of the image series (Figure 1f) shows the same NPs, after the oxygen supply has been stopped at the end of the measurement from above (top of Figure 1e). As it can be seen by the contrast of the WF image and corresponding profile underneath, the WF decreases in mean by $-0.1 \mathrm{eV}$, and in following images (not shown) by $\sim-0.3 \mathrm{eV}$. As explained further below, the WF increase and decrease can be assigned to a dissociative adsorption of oxygen on the (111) facets of the PdNPs and to a loss of oxygen, respectively.

Oxygen Exposure on G@PdNPs. In the following, we compare the oxygen adsorption characteristics of the as-prepared PdNPs with results obtained on G@PdNPs. Figure 2a and b show typical G@PdNPs after the graphene growth in ethylene (several hundreds of L) at temperatures between 670 and $740^{\circ} \mathrm{C}$. As described in Ref. [ 5], a single moiré pattern can be found on large and symmetrically shaped NPs (one perfect graphene sheet) whereas unsymmetrical NPs with a size below $\sim 30 \mathrm{~nm}$ exhibit several types of moiré patterns (graphene sheets) together with line defects on the NP's facets and probably also defects at the edges of the NPs. The WF of such PdNPs is strongly decreased by $\sim-1.4 \mathrm{eV}$ with respect to the WF of palladium ${ }^{6}$, moving the WF about $\sim-0.1 \mathrm{eV}$ below the WF of the HOPG surface, thus the NPs appear in a dark contrast in WF images, as it can be seen in the WF image of Figure 3a.

In Figure 3a-d, four successively recorded KPFM images are shown, which were selected from a series of 26 images. They present the same G@PdNPs on a time scale of 175 minutes. As before, the recording of one image needs 35 minutes with $t_{\text {line }}=4 \mathrm{sec}$, and, within an 

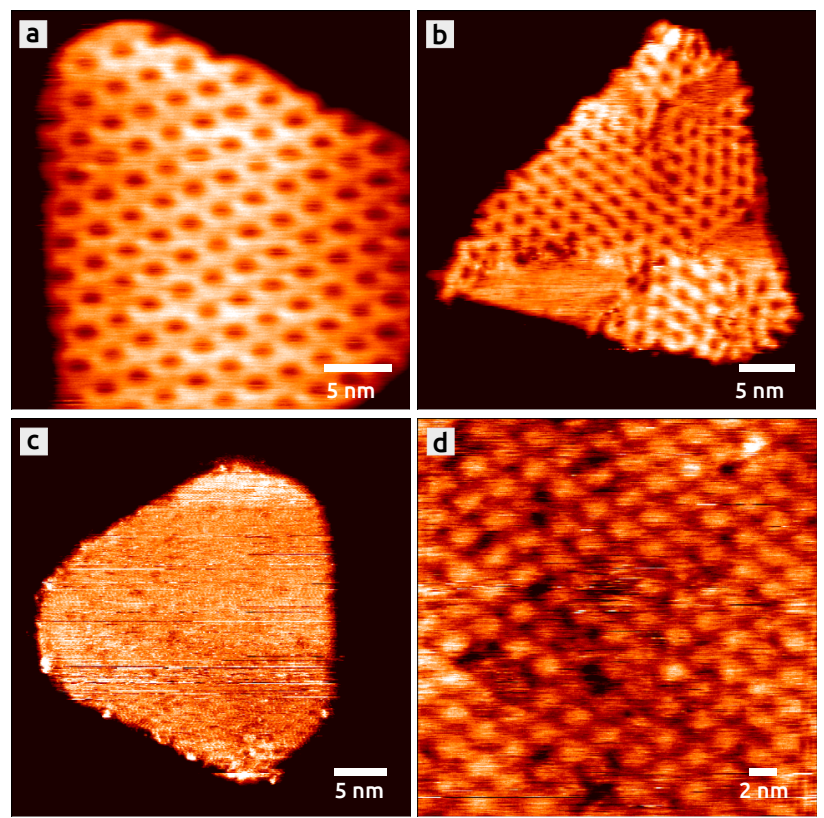

Figure 2: STM topography images of G@PdNPs. A single $2.52 \mathrm{~nm}$ large $(2 \sqrt{21} \times 2 \sqrt{21})$ R10.9 $(\mathrm{a})$ and a $1.92 \mathrm{~nm}$ large $(7 \times 7)$ - R21.8 $8^{\circ}$ moiré pattern (b) can be seen. (c,d) Top facet of a NP with a $2.47 \mathrm{~nm}$ large $(9 \times 9)$ - R0.0 $0^{\circ}$ moiré pattern, observed after an oxygen dosage. NP growth: $1.5 \mathrm{ML}$ at $475^{\circ} \mathrm{C}(\mathrm{a}, \mathrm{c}, \mathrm{d})$ and $2.0 \mathrm{ML}$ at $500^{\circ} \mathrm{C}(\mathrm{b})$, Graphene growth: $847 \mathrm{~L}$ of $\mathrm{C}_{2} \mathrm{H}_{4}\left(1 \times 10^{-6} \mathrm{mbar}\right)$ at $670{ }^{\circ} \mathrm{C}(\mathrm{a}, \mathrm{c}, \mathrm{d})$ and $900 \mathrm{~L}$ of $\mathrm{C}_{2} \mathrm{H}_{4}\left(2 \times 10^{-6} \mathrm{mbar}\right)$ at $735^{\circ} \mathrm{C}$ (b), STM parameters: $U_{\text {Bias }}=0.41$ (a), 0.09 (b) and $0.43 \mathrm{~V}(\mathrm{c}, \mathrm{d}), I=0.43$ (a), $0.42 \mathrm{nA}$ (b) and $0.37 \mathrm{nA}(\mathrm{c}, \mathrm{d}), v=3.3(\mathrm{a}), 2.0(\mathrm{~b}, \mathrm{c})$ and $3.0 \mathrm{~Hz}(\mathrm{~d})$.

image, the surface is scanned from the bottom to the top, with the blue and orange arrows marking the time line.

During the image acquisition, molecular oxygen is dosed in several steps on the NPs at room temperature: in the first measurement (Figure 3a), $5.5 \times 10^{-7}$ mbar of molecular oxygen is dosed into the UHV chamber (orange arrow). An important observation is that at this pressure, no WF changes are visible, unlike the case of the as-prepared NPs (compare with Figure $1 b)$. To observe any changes, the oxygen pressure is increased onto $1.5 \times 10^{-5}$ mbar (see Figure S3b in the Supporting Information), and even at this pressure, no immediate WF increase can be seen; the WF increases rather slowly such that after a few minutes, the WF of the NPs reaches the same WF of HOPG. To increase the speed of the WF change 


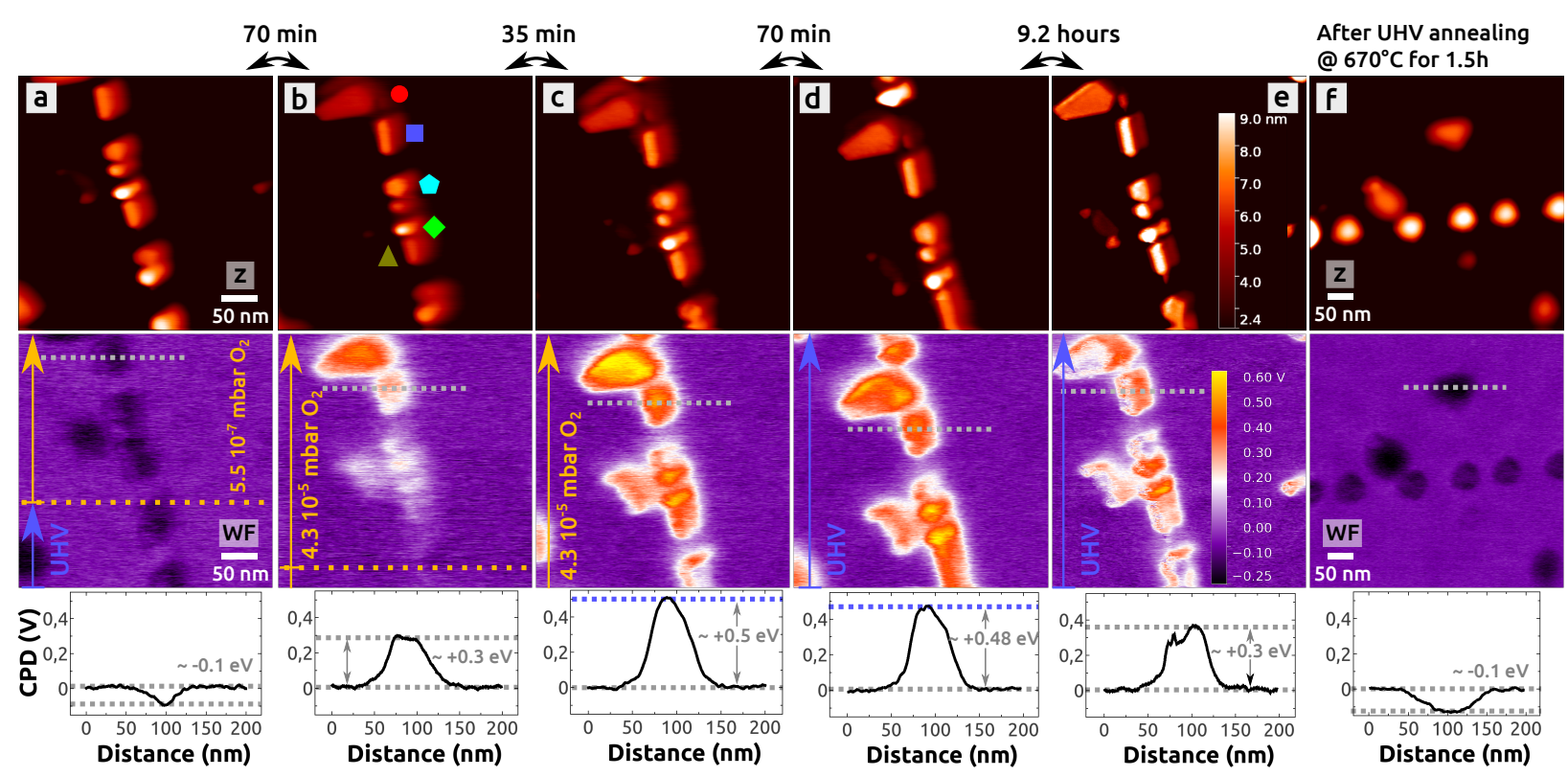

Figure 3: (a-e) Graphene encapsulated PdNPs imaged by KPFM during an exposure of $\mathrm{O}_{2}$ at room temperature $\left(620 \mathrm{~L} @ p_{\mathrm{O} 2}=5.5 \times 10^{-7} \operatorname{mbar}(\mathrm{a}), 2.2 \times 10^{4} \mathrm{~L} @ 1.5 \times 10^{-5} \mathrm{mbar}(\mathrm{b})\right.$ and $1.5 \times 10^{5} \mathrm{~L} @ 4.3 \times 10^{-5}$ mbar $(\mathrm{c})$, total dosage: $\left.1.7 \times 10^{5} \mathrm{~L}\right)$. One KPFM experiment is represented by a vertically arranged topography (top) and WF image (bottom), with a representative WF profile underneath, taken from the gray dotted line in the WF image. The profiles show the contrast for one and the same NP. The WF images have all the same color scale (See Figure S3 in the Supporting Information). The orange arrows show the $\mathrm{O}_{2}$ exposure whereas blue arrows correspond to the imaging in UHV (no $\mathrm{O}_{2}$ ). (f) Same sample after an annealing in UHV $\left(4 \times 10^{-10} \mathrm{mbar}\right)$ at $670^{\circ} \mathrm{C}$ during $1 \mathrm{~h} 20 \mathrm{~min}$. NP growth: $1.5 \mathrm{ML}$ at $475{ }^{\circ} \mathrm{C}$, Graphene growth: $847 \mathrm{~L}$ of $\mathrm{C}_{2} \mathrm{H}_{4}\left(1 \times 10^{-6} \mathrm{mbar}\right)$ at $670{ }^{\circ} \mathrm{C}$, KPFM parameters: $\triangle f=-9.5(\mathrm{a}),-10.6(\mathrm{~b}),-12.7$ (c), -13.6 (d), -13.7 (e) and $-15.3 \mathrm{~Hz}(\mathrm{~g})$, all: $v=0.5 \mathrm{~Hz}$, $U_{\mathrm{ac}}=500 \mathrm{mV}$ and $f_{\mathrm{ac}}=630 \mathrm{~Hz}$.

furthermore, the oxygen pressure is increased onto a value of $4.3 \times 10^{-5}$ mbar (Figure $3 \mathrm{~b}$ ). After some scanning lines, the WF is higher than the one of HOPG (white color) and increases further more by a few hundreds of meV. In the third image (Figure 3c), the pressure is kept constant and the WF increase saturates at a mean WF difference of $+0.5 \pm 0.1 \mathrm{eV}$ for all NPs, similar to the case of the as-prepared PdNPs from above.

The oxygen supply is stopped in the next image (Figure S3e in the Supporting Informa- 

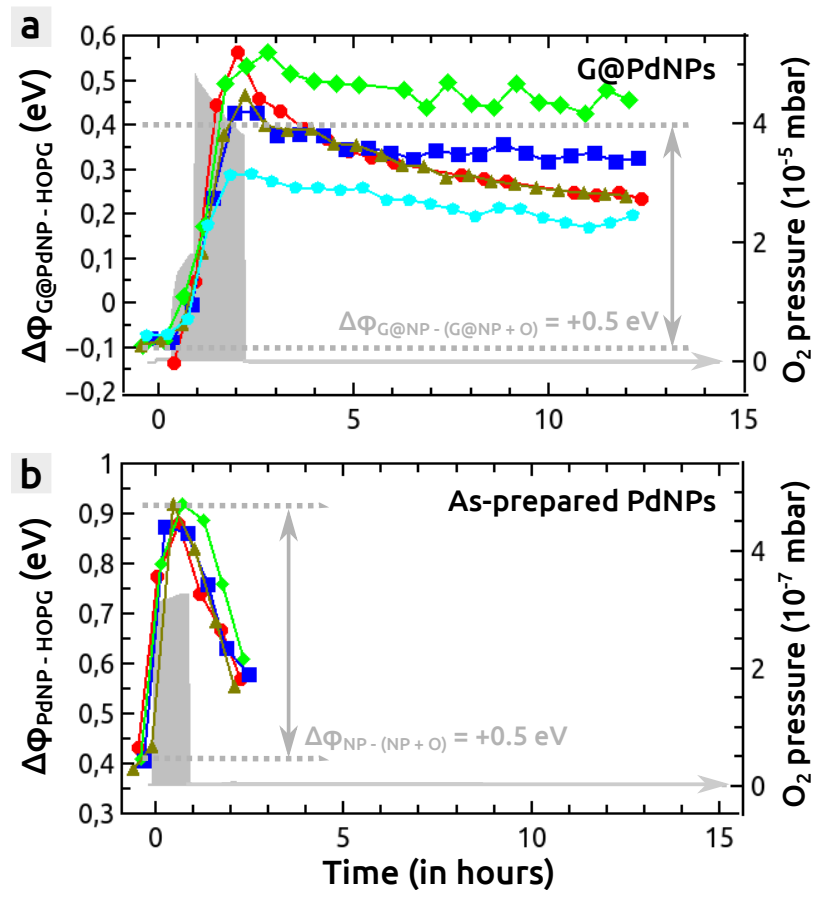

Figure 4: The temporal development of the WF difference $\triangle \phi$ between HOPG and the G@PdNPs from Figure 3 (a) and the as-prepared PdNPs from Figure 1 (b). The data was extracted at selected NPs, which are labeled in the topography images of Figure $3 \mathrm{~b}$ and Figure 1a. For the G@PdNPs (as-prepared PdNPs) the data is obtained from a series of 26 (6) images. Alongside the WF difference, the partial oxygen pressure is shown in form of the gray shaded region.

tion) and a slight decrease of the WF is detected. In the next following image (Figure 3d), the WF decrease measures only $\sim-0.06 \mathrm{eV}$, about 70 minutes after the oxygen supply has been stopped. Figure 3e shows the same NPs 10 hours after: although the WF difference has decreased onto $+0.3 \pm 0.1 \mathrm{eV}$, the NPs still exhibit a strong WF difference with the HOPG surface. In Figure 4a, the temporal development of the NP's WF is shown for some selected NPs (see blue dots in the topography image of Figure $3 \mathrm{~b}$ ), from the start of the oxygen dosage until 13 hours after: when the oxygen supply is closed, the WF decreases slightly but remains stable for many hours. This small WF decrease contrasts the situation of the as-prepared PdNPs, where the WF difference decreases much faster (see Figure 4b).

After a second oxygen adsorption experiment on the same NPs (see Figure $\mathrm{S} 4$ in the 
Supporting Information), STM has been used to verify if the graphene on top of the NPs is still intact. The two STM topography images in Figure 2c and d clearly show, that the graphene is still intact right after a dosage of oxygen: typical perfect moiré patterns can be seen, with a corrugation that is comparable with G@PdNPs, which are not dosed with oxygen ${ }^{5,6}$. Unfortunately, the atomic resolution could not be obtained to observe possible oxygen atoms underneath the graphene, as it was done in Ref. [ 30].

The very last preparation step consists of a post-annealing of the sample in UHV $\left(4 \times 10^{-10}\right.$ mbar) at $670{ }^{\circ} \mathrm{C}$ (Figure 3f). The initial very low WF of G@PdNPs is recovered (compare with Figure 3a), which can be seen by the typical dark contrast that corresponds to a WF difference of $\sim 0.1 \mathrm{eV}$ between the NPs and HOPG.

Density Functional Theory Calculations. To elucidate the origin of the observed changes in the WF, we use DFT calculations and explore a range of representative configurations of oxygen on $\operatorname{Pd}(111)$ and $\mathrm{Pd}_{6} \mathrm{C}(111)$ as well as of oxygen intercalated between graphene and the two surfaces. We consider two graphene configurations, namely the frequently observed $\sqrt{3} \times \sqrt{3}$ and $5 \times 5$ graphene structures ${ }^{5,6}$. The calculations are performed with surface cells of $(\sqrt{3} \times \sqrt{3}),(2 \sqrt{3} \times 2 \sqrt{3})$ or $(5 \times 5)$. The oxygen atoms at a given coverage are evenly distributed for each surface cell. Note that due to the special cell geometry, the distribution of the oxygen atoms may not reflect the experimentally observed structures. For example, at $0.25 \mathrm{ML}$ coverage, the measured structure is a $(2 \times 2)$ structure $^{43-47}$. Note also that we merely focus on the trends in the WF changes when oxygen is adsorbed on the surface because of the unknown coverage in the experiments. The DFT results are summarized in Table 1 and the corresponding structures are shown in Figure S7 of the Supporting Information.

Palladium is an fcc metal with an experimental lattice constant of $a_{\mathrm{Pd}, \mathrm{lit}}=3.89 \pm 0.01 \AA$ (see Table S3 in the Supporting Information). We calculate the lattice constant to be $3.87 \AA$. The slight underestimation by LDA is consistent with previous reports ${ }^{48}$. For the WF for the pristine $\mathrm{Pd}(111)$ surface, a value of $5.70 \mathrm{eV}$ is obtained. This value is consistent with previous 
Table 1: DFT results for the WF (in eV) for different oxygen coverages $(\rho)$ on $\operatorname{Pd}(111)$ and $\operatorname{Pd}_{6} \mathrm{C}(111)$, either with or without graphene $(\operatorname{Gr} \sqrt{3} \times \sqrt{3}$ and $\operatorname{Gr} 5 \times 5)$. The WF change $\triangle \mathrm{WF}$ (in $\mathrm{eV}$ ) induced by oxygen is also reported. For graphene on $\operatorname{Pd}(111)$ and $\operatorname{Pd}_{6} \mathrm{C}(111)$, the mean distance $d$ (in $\AA$ ) between graphene and the first atomic Pd surface layer is given.

\begin{tabular}{|c|c|c|c|c|c|c|c|c|c|c|c|}
\hline$\rho$ & $\mathrm{WF}$ & $\triangle \mathrm{WF} \quad d$ & $\mathrm{WF}$ & $\triangle \mathrm{WF}$ & $d$ & WF & $\triangle \mathrm{WF}$ & $d$ & $\mathrm{WF}$ & $\triangle \mathrm{WF}$ & $d$ \\
\hline & \multicolumn{2}{|r|}{$\operatorname{Pd}(111)$} & \multicolumn{3}{|c|}{$\operatorname{Gr} \sqrt{3} \times \sqrt{3} / \operatorname{Pd}(111)$} & \multicolumn{3}{|c|}{$\mathrm{Pd}_{6} \mathrm{C}(111)$} & \multicolumn{3}{|c|}{$\operatorname{Gr} \sqrt{3} \times \sqrt{3} / \mathrm{Pd}_{6} \mathrm{C}(111)$} \\
\hline 0 & 5.70 & $0 \quad /$ & 4.68 & 0 & 3.00 & 5.54 & 0 & / & 4.68 & 0 & 2.92 \\
\hline $1 / 12(0.083)$ & 5.94 & $+0.24 \quad /$ & 4.81 & +0.13 & 2.90 & 5.82 & +0.28 & / & 5.04 & +0.36 & 3.28 \\
\hline $2 / 12(0.167)$ & 6.18 & $+0.48 \quad /$ & 5.02 & +0.34 & 3.32 & 6.09 & +0.55 & / & 5.23 & +0.55 & 3.52 \\
\hline $3 / 12(0.250)$ & 6.39 & $+0.69 \quad /$ & 5.14 & +0.46 & 3.57 & 6.38 & +0.84 & / & 5.33 & +0.65 & 3.69 \\
\hline $4 / 12(0.333)$ & 6.62 & $+0.92 \quad /$ & 5.19 & +0.51 & 3.79 & 6.65 & +1.11 & / & 5.39 & +0.71 & 3.77 \\
\hline & & & \multicolumn{3}{|c|}{ Gr $5 \times 5 / \operatorname{Pd}(111)$} & & & & & & \\
\hline 0 & & & 4.55 & 0 & 2.62 & & & & & & \\
\hline $1 / 25(0.040)$ & & & 4.83 & +0.28 & 2.58 & & & & & & \\
\hline $2 / 25(0.080)$ & & & 4.79 & +0.24 & 2.50 & & & & & & \\
\hline $2 / 25(0.080)$ & & & 5.10 & +0.55 & 3.22 & & & & & & \\
\hline $6 / 25(0.024)$ & & & 5.40 & +0.85 & 3.70 & & & & & & \\
\hline
\end{tabular}

computational reports and agree well with the experimental value of $\phi_{\mathrm{Pd} \text {,lit }}=5.6 \pm 0.1 \mathrm{eV}$ (see Table S2 in the Supporting Information).

Molecular oxygen adsorbs dissociatively on $\operatorname{Pd}(111)$ and PdNPs, and the oxygen atoms occupy preferably fcc hollow sites ${ }^{49}$. In the following, we only consider fcc hollow sites, also when oxygen is intercalated between the graphene sheet and the palladium surface. We calculate the WF of the $\operatorname{Pd}(111)$ surface for four different oxygen coverages, namely $1 / 12$, 2/12,3/12 and 4/12 monolayer (ML). One ML is defined as the the number of surface atoms whereas max. 12 oxygen atoms can be present in the $2 \sqrt{3} \times 2 \sqrt{3}$ cell. The $3 / 12$ oxygen coverage is shown in Figure 5a whereas all the other coverages are shown in Figure S7 of the Supporting Information.

With increasing coverage (see Table 1), the WF of the Pd(111) surface increases monotonously, with a WF shift of $+0.69 \mathrm{eV}$ at a $3 / 12 \mathrm{ML}(0.25 \mathrm{ML})$ coverage and a maximum shift of 
$+0.92 \mathrm{eV}$ at $4 / 12 \mathrm{ML}(0.33 \mathrm{ML})$, which is in qualitative agreement with previous reports (e.g., $+0.5 \mathrm{eV}$ for a $2 \times 2$ related $0.25 \mathrm{ML}$ coverage ${ }^{49,50}$ ). Note that we also modeled the 0.25 ML $2 \times 2$ structure described in literature ${ }^{43-47}$ (see Figure S8 in the Supporting Information). For this structure, we obtain the same WF as above (3/12 ML coverage), i.e., the WF does not depend sensitively on the detailed arrangement of the surface oxygen atoms. The monotonous increase of the WF is owing to the increasing density of the oxygen atoms, which carry a negative charge and create a net surface dipole oriented towards the surface, overall increasing the WF of the metal.

A monotonous WF increase is observed also when increasing the coverage of intercalated oxygen atoms (Figure 5b). In comparison to oxygen on $\mathrm{Pd}(111)$, the WF shifts are smaller being +0.46 and $+0.51 \mathrm{eV}$ at $3 / 12 \mathrm{ML}(0.25 \mathrm{ML})$ and 4/12 ML (0.33 ML) coverage, respectively. With respect to the mean distance $d$ between graphene and $\operatorname{Pd}(111)$, the value first decreases slightly by $-0.1 \AA$ but increases monotonously by up to $+0.8 \AA$ at a $4 / 12 \mathrm{ML}$ coverage. A similar increase of the mean distance upon oxygen intercalation was observed on graphene $/ \operatorname{Rh}(111)^{51}$. Note that an increase of the distance leads to an increase of the surface $\mathrm{WF}^{6}$ because it reduces the charge density overlap and thereby the electrostatic compression owing to the Pauli principle. Overall, the WF increase upon oxygen intercalation clearly demonstrates that the surface WF is not determined by graphene alone but rather by three contributions: i) the metal WF, ii) the surface dipole layer induced by the oxygen atoms and iii) the electrostatic compression effect induced by graphene.

We now consider the $\mathrm{Pd}_{6} \mathrm{C}(111)$ surface, which represents the (111) facets of those PdNPs grown at relative high temperatures on HOPG and therefore contain subsurface carbon ${ }^{6}$. We consider the same oxygen coverages and adsorption sites as for the pure palladium case above. Oxygen is adsorbed on $\mathrm{Pd}_{6} \mathrm{C}(111)$ (Figure 5c) or intercalated between the graphene wall and $\mathrm{Pd}_{6} \mathrm{C}(111)$ (Figure 5d). As on $\mathrm{Pd}(111)$, the WF shifts are all positive and the WF is monotonously increasing with increasing coverage. The WF shifts for $\mathrm{O} / \mathrm{Pd}{ }_{6} \mathrm{C}(111)$ are larger than for intercalated oxygen. Furthermore, the mean graphene- $\mathrm{Pd}_{6} \mathrm{C}(111)$ distance 


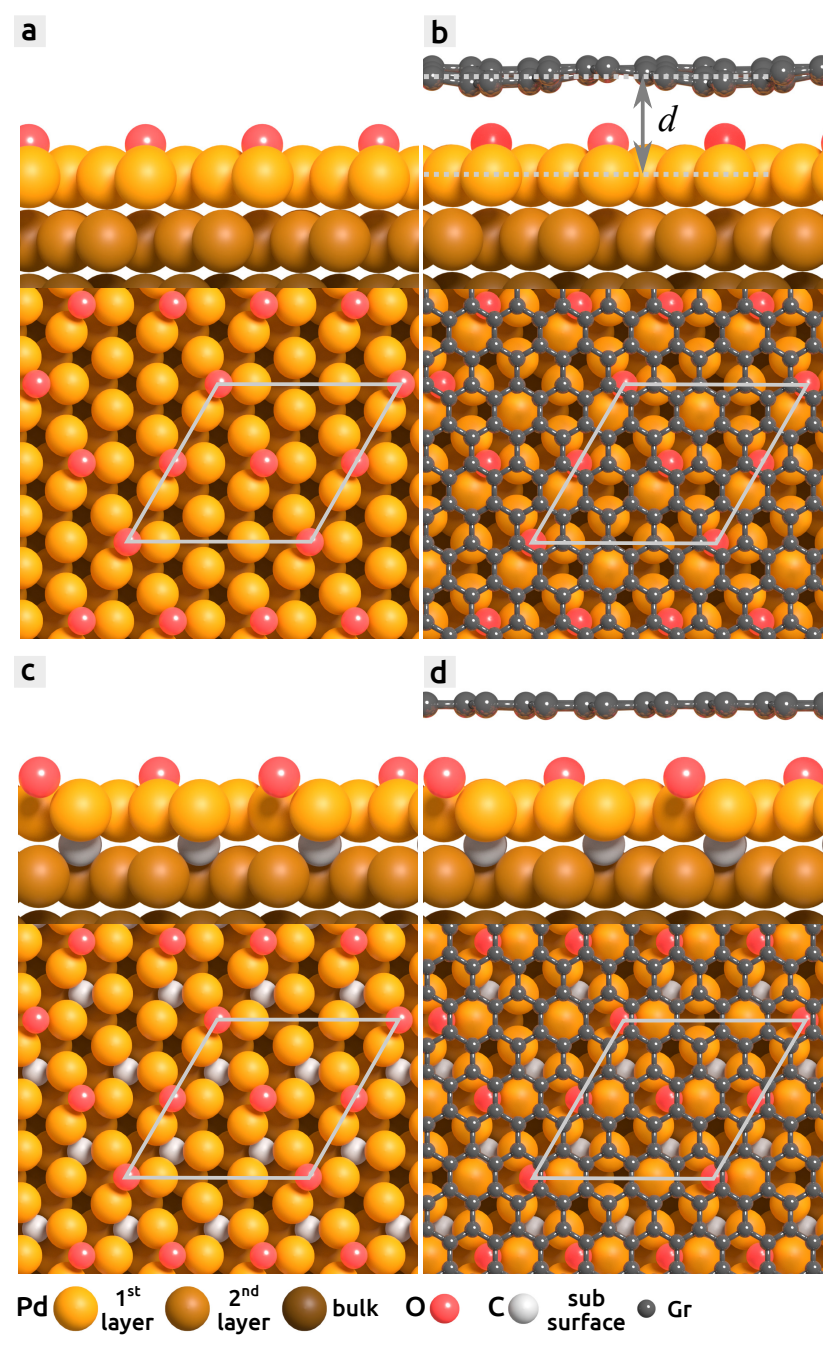

Figure 5: Atomic O with a coverage of 3/12 ML (0.25 ML) on different surfaces: $\mathrm{O}$ adsorbed on $\operatorname{Pd}(111)$ (a), O intercalated between graphene $\sqrt{3} \times \sqrt{3}$ and $\operatorname{Pd}(111)$, $\mathrm{O}$ adsorbed on $\mathrm{Pd}_{6} \mathrm{C}(111)$ (c), and $\mathrm{O}$ intercalated between graphene $\sqrt{3} \times \sqrt{3}$ and $\operatorname{Pd}_{6} \mathrm{C}(111)(\mathrm{d})$. The structures are shown in side and top views. The light gray rhomb shows the $2 \sqrt{3} \times 2 \sqrt{3}$ surface cell used for the calculations.

increases with increasing coverage. In comparison to $\mathrm{Pd}(111)$, the WF shifts are somewhat larger, in mean by 0.1 to $0.2 \mathrm{eV}$. Overall, $\mathrm{Pd}_{6} \mathrm{C}(111)$ behaves similar to $\mathrm{Pd}(111)$.

To understand the dependence of the induced WF changes on the graphene configuration, we consider the $5 \times 5$ graphene structure on $\mathrm{Pd}(111)$ (Figure 6a), which is regularly observed by $\mathrm{STM}^{5,6}$. Because the size of this surface cell is considerably larger than the $\sqrt{3} \times \sqrt{3}$ 


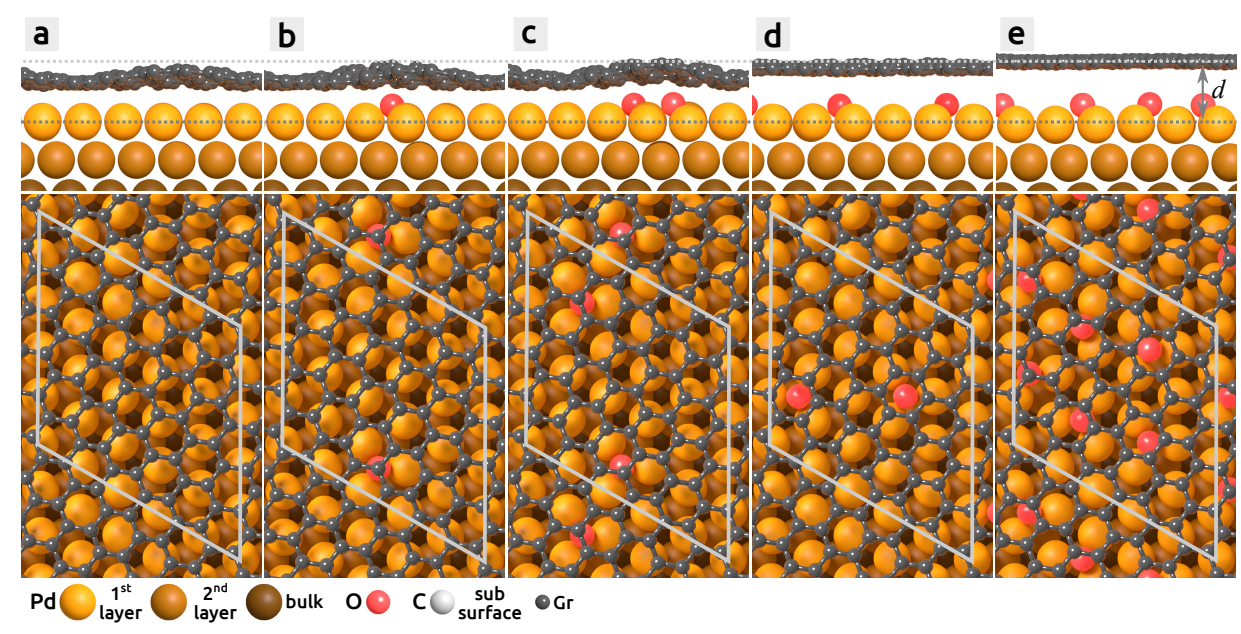

Figure 6: Atomic $\mathrm{O}$ intercalated between $\mathrm{Pd}(111)$ and the $5 \times 5$ graphene structure for different O coverages: 0 (a), 1/25 (b), 2/25 (c,d) and 6/25 ML (e). Images (c) and (d) show the same $\mathrm{O}$ coverage but for two different $\mathrm{O}$ distributions. The structures are shown in side and top views. The light gray rhomb shows the surface cell of the $5 \times 5$ graphene structure used for the calculations.

structure $(1.37 \mathrm{~nm}$ versus $0.48 \mathrm{~nm}$ side length) there are many possibilities to place oxygen atoms. Starting with a coverage of $1 / 25 \mathrm{ML}(0.04 \mathrm{ML})$ in the configuration shown in Figure $6 \mathrm{~b}$, the WF shift is $+0.28 \mathrm{eV}$ large. If the coverage is doubled onto $2 / 25 \mathrm{ML}(0.08 \mathrm{ML})$, the two independent O configurations (Figure 6c and d) yield clearly different WF shifts: in the first case, the graphene sheet is closer to the $\operatorname{Pd}(111)$ surface (Figure 6c) whereas in the second case the graphene is $0.72 \AA$ farer away from the surface (Figure $6 \mathrm{~d}$ ). As a result of the electrostatic compression effect ${ }^{6}$, the WF shift is smaller in the first case $(+0.24 \mathrm{eV})$ than in the second one $(+0.55 \mathrm{eV})$.

When the coverage is increased onto 6/25 (0.24 ML) (Figure6e), we obtain a WF shift of $+0.85 \mathrm{eV}$, which is larger than the value of the $\sqrt{3} \times \sqrt{3}$ graphene structure at $3 / 12$ $(0.25 \mathrm{ML})$ coverage $(+0.46 \mathrm{eV})$. However, the values are still comparable and we expect that the WF shift for a $0.25 \mathrm{ML}$ oxygen coverage is well below $1 \mathrm{eV}$, independent on the graphene orientation on $\operatorname{Pd}(111)$ and $\operatorname{Pd}_{6} \mathrm{C}(111)$.

Alongside the distance dependence of the WF, there also is a considerable buckling of the 
graphene, which, in particular, is present in the absence of intercalated oxygen (Figure 6a). For a low oxygen concentration $(1 / 25 \mathrm{ML}$, Figure $6 \mathrm{~b})$, the oxygen atoms do not influence the distance between graphene and $\operatorname{Pd}(111)$ surface if they are placed at positions where the distance is largest. The same applies for a slightly larger coverage (Figure 6c), however, some oxygen atoms start to separate the graphene sheet from the metal where the distance to the surface is relatively close. If for the same coverage, the oxygen atoms are differently distributed, e.g., at sites where the graphene distance is relatively close, the adsorption leads to an increased metal-graphene distance (Figure 6d). Finally, with increasing oxygen coverage, the oxygen atoms displace more and more the graphene sheet from the surface (Figure 6e). Similar phenomena were suggested before ${ }^{52}$ and observed by DFT on $\operatorname{Rh}(111)^{51}$. At relatively large mean distances, which are obtained at in particular high oxygen coverages (e.g., at 6/25 (0.24 ML) coverage, Figure 6e), the buckling is less pronounced or even not present, and graphene adopts a more free-standing character. A similar though smaller buckling can be also found for the $\sqrt{3} \times \sqrt{3}$ graphene structure at low oxygen coverages (see Figure 5b).

\section{DISCUSSION}

Mechanisms of the Oxygen Adsorption on As-Prepared PdNPs. It is well known that molecular oxygen dissociatively adsorbs on $\mathrm{Pd}(111)$ single crystal surfaces at room temperature $(\mathrm{RT})^{43,44}$. The dissociative adsorption of oxygen was extensively studied by, e.g., LEED ${ }^{43-46}$, photoemission spectroscopy ${ }^{43-45}$, Auger spectroscopy ${ }^{44-46}$, temperature programmed desorption (TPD) ${ }^{46}$ and $\mathrm{STM}^{46,53,54}$ and also described in detail by several DFT works $^{49,50,55,56}$. In the following, we only consider the adsorption of atomic oxygen on the

surface. Surface oxides ${ }^{46,57,58}$ and subsurface oxygen ${ }^{47,59}$ appear at higher temperatures and pressures and can therefore be neglected for the following discussion.

Our and previous DFT results ${ }^{50}$ show that a charge transfer from palladium to oxygen 
takes place creating a surface dipole towards the surface, which the latter increases the surface WF. The WF change depends sensitively on the oxygen coverage, with values ranging from roughly +0.2 to $+1.0 \mathrm{eV}$ for $1 / 12$ to $1 / 3 \mathrm{ML}$ oxygen, respectively (see Table 1 ). Although we could not obtain the atomic resolution on the oxygen dosed NPs by STM like in Ref. [60], we strongly anticipate that the oxygen atoms form the well-known $2 \times 2$ structure, which was frequently observed by LEED ${ }^{43-47}$ and RT STM ${ }^{46}$ : initially, this structure was assigned to an oxygen coverage of $1 / 4 \mathrm{ML}^{43}$, which was questioned by a following work that found rather a $1 / 2 \mathrm{ML}$ coverage ${ }^{44}$. However, the $2 \times 2$ related $1 / 4 \mathrm{ML}$ coverage got confirmed by more recent work ${ }^{45,46}$. The $2 \times 2$ structure starts to appear after a few Langmuir ${ }^{43,44,46}$ and is completed after a few tens of Langmuir ${ }^{46}$. The structure is stable after an oxygen dosage of $10^{5} \mathrm{~L}^{44}$, which is much higher than the one used for the experiments shown in Figure $1(771 \mathrm{~L})$. No other oxygen structures appear at RT and during continued oxygen dosages $^{46}$. Note that we exclude a possible $\sqrt{3} \times \sqrt{3} \mathrm{R} 30^{\circ}$ structure, which is supposed to coexist with the $2 \times 2$ structure at RT up to a dosage of $500 \mathrm{~L}$ and which is stabilized by CO and hydrogen ${ }^{43}$. We also exclude a $1 \times 1$ structure, which seem to appear only after very high oxygen dosages $\left(>10^{5} \mathrm{~L}\right)^{44}$.

Overall, we can conclude that in our experiments, the oxygen coverage was at around 1/4 ML. Comparing the experimental WF increase of $+0.6 \mathrm{eV}$ (Figure 1e) with the values obtained from theory (see Table 1) we find a good quantitative agreement at 3/12 ML coverage where theory predicts a WF change of $+0.69 \mathrm{eV}$ for $\mathrm{Pd}(111)$ and $+0.84 \mathrm{eV}$ for $\mathrm{Pd}_{6} \mathrm{C}(111)$. A quantitative agreement is also obtained when considering work done with the macroscopic Kelvin probe technique ${ }^{34}$ : a saturation of the WF increase was observed after a few Langmuir $\left(p_{\mathrm{O} 2}=2.7 \times 10^{-8}\right.$ mbar $)$ at RT, with a maximum WF change of +0.8 to $+0.9 \mathrm{eV}$.

Nevertheless, our experimental values of the WF shift are a bit too small, by 0.1 to $0.2 \mathrm{eV}$, which might be due to a tip-NP convolution effect ${ }^{61-64}$ : because the size of the tip apex is comparable to the size of the NP, the convolution effect reduces the relative WF difference between the PdNPs and HOPG, with a possible but so far unknown impact on 
also WF changes induced by oxygen. Because the tip dimension and tip-surface distance, which strongly influence the convolution, are unknown in our experiments, it is difficult to estimate the relative deviation from the true values of WF differences. In future, the influence of the convolution and other possible effects need to be studied in more detail.

The WF decrease in Figure 1f and Figure 4b, which appears after an oxygen adsorption experiment is stopped, can be related to a loss of atomic oxygen on the (111) facets of the NPs. In principle, when the oxygen supply is stopped there is no adsorption/desorption equilibrium anymore and only oxygen desorption may appear. However, this is contrary to the general finding that at room temperature, atomic oxygen should be quite stable on $\operatorname{Pd}(111)$ : the binding energy is between -1.3 and $-1.5 \mathrm{eV}^{50,55}$. We therefore believe that rather a chemical reaction with molecules from the residual UHV gas removes the oxygen because with the UHV system used here, best UHV conditions $\left(\sim 10^{-10} \mathrm{mbar}\right)$ are not immediately obtained when stopping the oxygen supply: after closing the oxygen valve, the residual pressure of the UHV first immediately decreases onto $\sim 10^{-8}$ mbar, decreases then within a few minutes onto $2 \times 10^{-9}$ mbar and decreases furthermore but very slowly within a few hours. Although we cannot exclude a reaction between the adsorbed oxygen with residual $\mathrm{CO}^{65}$, we rather believe that it is due to a reaction with hydrogen: with hydrogen being the largest contributor to the residual gas of the UHV, the surface is dosed with $\sim 3 \mathrm{~L}$ of hydrogen during the image acquisition of Figure 1f. Therefore it could be that hydrogen reacts off a part of the oxygen on the PdNPs, presumably forming water at $\mathrm{RT}^{43,66-68}$.

Comparison with Oxygen Adsorption on G@PdNPs. In comparison to the asprepared PdNPs from above, similar oxygen adsorption phenomena can be observed on the G@PdNPs (Figure 3). However, there are three main differences: (a) a much higher pressure $(\sim 100 \times)$ is needed to observe a similar saturated WF increase at the G@PdNPs, (b) the WF increase seems to need more time at G@PdNPs, unlike the immediate increase on as-prepared PdNPs and (c) the oxygen removal is much slower in the G@PdNP case.

Because oxygen is generally not adsorbing on graphene, the WF increase observed in 
our experiments (Figure 3) can be assigned to oxygen, which is intercalated between the graphene wall and the facets of the NPs. This is quite similar with respect to oxygen adsorption experiments conducted on single crystal surfaces like $\operatorname{Ir}(111)^{30,69}, \operatorname{Pt}(111)^{70}$ and $\mathrm{Ru}(0001)^{52,71}$. The WF contrast at the NPs is relatively homogeneous, which shows that oxygen is evenly distributed on the facets. An intercalation of oxygen is strongly supported by our DFT calculations, which show that the WF increases when oxygen is intercalated between graphene and the NP's facets (Table 1). As in the case of the as-prepared PdNPs, the WF increases with increasing oxygen density (Table 1), and for a coverage between $1 / 4$ and $1 / 3 \mathrm{ML}$ a WF shift between rougly +0.5 and $+0.7 \mathrm{eV}$ is found on $\operatorname{Pd}(111)$ and $\mathrm{Pd}_{6} \mathrm{C}(111)$, which is in quantitative agreement with our experimental observations $(+0.5 \mathrm{eV})$. Although we could image a graphene moiré pattern (Figure $2 \mathrm{~d}$ ) we could not obtain the atomic resolution, which normally can be used to estimate the density of the oxygen atoms ${ }^{30}$. However, we can assume similar oxygen coverages as in the case of the as-prepared PdNPs from above due to the following reasons: (a) increasing the coverage requires higher pressures which would be quite limited here due to the presence of the nanocontainer formed by graphene and the NP's facets, (b) on, e.g., $\mathrm{Rh}(111)$ the coverage of intercalated oxygen resembles much the one of oxygen on the pristine surface ${ }^{51}$.

Because perfect graphene is impermeable for all gases ${ }^{72}$ and does not adsorb ${ }^{42}$ or dissociate $^{73}$ oxygen at RT, the oxygen needs to pass defects of the graphene to access the Pd facets. Indeed, defects in the graphene could be recently observed on G@PdNPs by STM ${ }^{5,6}$ and can be also expected to be present at the edges formed by the NP's facets. Molecular oxygen certainly dissociates with the help of those active $\mathrm{Pd}$ atoms that are not covered by graphene, i.e., below the graphene defects $\left(\mathrm{Pd}_{\mathrm{a}}\right.$ atoms) such that the oxygen atoms can then adsorb and diffuse on the facets and below the graphene sheet ${ }^{51,74}$. The dissociation and adsorption process of oxygen is such that the graphene remains intact, which is because we still observe the moiré pattern in the presence of oxygen by STM (Figure 2c and d).

The oxygen intercalation via defects in the graphene explains the observation that a high 
oxygen partial pressure is needed to adsorb oxygen inside the nanocontainer formed by the graphene and the NP's facets: because only the small amount of $\mathrm{Pd}_{\mathrm{a}}$ atoms contributes to the oxygen adsorption, the number of impinging oxygen molecules (pressure) needs to be increased at the $\mathrm{Pd}_{\mathrm{a}}$ atoms such that the same oxygen adsorption can be observed within the same time, during which observations were done on the as-prepared PdNPs. Probably, the process of oxygen dissociation, adsorption and diffusion into the nanocontainer is kinetically limited, which explains why the overall adsorption needs a longer time compared to the case where adsorption takes place instantaneously on the facets of as-prepared PdNPs. Note that a quite similar phenomena is observed on crystalline metal surfaces where, e.g., on $\operatorname{Ir}(111)$ the oxygen dosage needs to be more than a magnitude higher (up to $10^{5} \mathrm{~L}^{30}$ ) than what is necessary to reach saturation coverage on pristine $\operatorname{Ir}(111)^{69}$.

If it is assumed that the adsorption is proportional to the number of the $\mathrm{Pd}_{\mathrm{a}}$ atoms, the relative pressure increase of two orders of magnitude can then be calculated from the density of the $\mathrm{Pd}_{\mathrm{a}}$ atoms below the defects and the overall density of palladium atoms forming the entire top (111) facet (see Figure S5 and S6 in the Supporting Information). If it is assumed for simplicity, that defects in the graphene can be found in particular at the edges of the NPs, a factor of 100 is indeed obtained for the case, that each second palladium atom at the edge takes place in the dissociation process (see Supporting Information).

The WF decrease in Figure 3d and e and in Figure 4a, which appears after an oxygen adsorption experiment is stopped, can be assigned again to a loss of atomic oxygen in the nanocontainer. As for the as-prepared NP, we believe that the very slow WF reduction is due to a reaction with hydrogen from the residual gas of the UHV. Because hydrogen as well as the final product molecule (e.g., water) need to pass the graphene defects, the process of oxygen removal is mass-transfer limited and slow. Indeed, the WF reduction is much slower (Figure 4a) compared to the one observed at the as-prepared PdNPs (Figure 4b). In other words, once oxygen is adsorbed inside the nanocontainer, it is more or less well passivated from a gaseous environment. 
The oxygen removal can be much accelerated, when annealing the G@PdNPs at very high temperatures in UHV: as shown before ${ }^{32}$, adsorbed oxygen on PdNPs starts to recombine to molecular oxygen at temperatures higher than $\sim 600^{\circ} \mathrm{C}$ desorbing from the NPs. This seems to be the same here because the initially low WF of the G@PdNPs is recovered after an anneal at $670{ }^{\circ} \mathrm{C}$ (Figure 3f) - a clear signature that oxygen has left the nanocontainer.

\section{CONCLUSIONS}

Thanks to the high lateral resolution at the nanometer scale and the high resolution in work function (WF) of Kelvin probe force microscopy (KPFM), adsorption and desorption phenomena of atomic and molecular species can be observed on single nanoparticles (NP) by measuring changes of the NP's WF. We exemplify this with oxygen adsorption KPFM experiments conducted at room-temperature and under UHV conditions, at as-prepared palladium NPs (PdNPs) and graphene encapsulated palladium NPs (G@PdNPs) supported on highly oriented pyrolytic graphite (HOPG). Our results deliver unambiguously the direct proof for the adsorption of oxygen under the graphene cover of G@PdNPs.

When a few Langmuir (L) of molecular oxygen are dosed on PdNPs, oxygen adsorbs dissociatively and immediately increases the NP's WF by up to $+0.6 \mathrm{eV}$, which is in agreement with our DFT calculations. A similar WF increase is observed at G@PdNPs, which clearly shows that oxygen is intercalated in the nanocontainer formed by graphene and the NP's facets. However, the oxygen adsorption and desorption characteristics are quite different comparing PdNPs with G@PdNPs: (a) a very high oxygen partial pressure is needed ( $\sim 10^{-5}$ mbar) for G@PdNPs to observe the intercalation of oxygen in the nanocontainer. In comparison, a two orders of magnitude smaller pressure is needed for PdNPs $\left(\sim 10^{-7}\right.$ mbar $)$.

(b) The oxygen adsorption on G@PdNPs needs more time in comparison to the immediate oxygen adsorption on PdNPs. (c) On G@PdNPs, oxygen is more protected from reactions with the residual gas of the UHV in comparison to PdNPs. We conclude that there is a 
limited facility for molecules to enter and leave the nanocontainer, which is simply due to the fact that the molecules need to pass defects of the graphene.

With respect to the oxygen intercalation, atomic resolution STM or nc-AFM can be used in future to observe the intercalated oxygen underneath graphene and to quantify also the defects in the graphene. STM ${ }^{71}$ or AFM spectroscopy ${ }^{75}$ could further check weather or not the graphene is decoupled and exhibits same electronic properties like free-standing graphene. The general and most important motivation of our work is to accomplish adsorption and desorption KPFM experiments also with other reactant molecules (e.g., hydrogen, CO and water) and other NP materials, which opens large perspectives to study phenomena related to catalysis and surface chemistry at the single NP level.

\section{ACKNOWLEDGMENTS}

Support from the Agence Nationale de la Recherche and the Deutsche Forschungsgemeinschaft (ANR, DFG) through project REACTIVITY (grant ANR-17-CE09-0045) is gratefully acknowledged. H.G. acknowledges support from the Swedish Reserach Council (2016-05234). The calculations were performed at C3SE (Göteborg) via an SNIC grant. C.B. acknowledges J. Murgia and P. Bindzi for maintainance work at the UHV chamber.

\section{ASSOCIATED CONTENT}

Supporting Information. Details about the sample preparation, UHV techniques (STM, nc-AFM and KPFM), image analysis, density functional theory (DFT) calculations, choice of WF values from literature, and supporting experiments and calculations (PDF)

\section{AUTHOR INFORMATION}

\section{Corresponding Author}


E-mail: barth@cinam.univ-mrs.fr

\section{ORCID}

Henrik Grönbeck: 0000-0002-8709-2889

Clemens Barth: 0000-0003-4250-4533

\section{Author Contributions}

C. B. conducted the experimental work whereas H. G. performed the calculations. C. B. and

H. G. wrote the manuscript.

\section{Funding Sources}

Agence Nationale de la Recherche (ANR-17-CE09-0045, project REACTIVITY). Swedish Research Council (2016-05234).

\section{Notes}

The authors declare no competing financial interests.

\section{References}

1. Peng, Z.; Somodi, F.; Helveg, S.; Kisielowski, C.; Specht, P.; Bell, A. T. High-Resolution in Situ and Ex Situ TEM Studies on Graphene Formation and Growth on Pt Nanoparticles. J. Catal. 2012, 286, 22-29.

2. Kim, H.; Robertson, A. W.; Kim, S. O.; Kim, J. M.; Warner, J. H. Resilient High Catalytic Performance of Platinum Nanocatalysts With Porous Graphene Envelope. ACS Nano 2015, 9, 5947-5957.

3. Gao, L.; Fu, Q.; Li, J.; Qu, Z.; Bao, X. Enhanced CO Oxidation Reaction Over Pt Nanoparticles Covered With Ultrathin Graphitic Layers. Carbon 2016, 101, 324-330.

4. Sengar, S. K.; Mehta, B. R.; Kumar, R.; Singh, V. In-Flight Gas Phase Growth of Metal/Multi Layer Graphene Core Shell Nanoparticles With Controllable Sizes. Sci. Rep. 2013, 3, 2814. 
5. Barth, C. Carbon Precursor Structures and Graphene on Palladium Nanoparticles. J. Phys. Chem. C 2018, 122, 522-529.

6. Grönbeck, H.; Barth, C. Revealing Carbon Phenomena at Palladium Nanoparticles by Analyzing the Work Function. J. Phys. Chem. C 2019, 123, 4360-4370.

7. Li, Y.; Chopra, N. Graphene Encapsulated Gold Nanoparticle-Quantum Dot Heterostructures and Their Electrochemical Characterization. Appl. Surf. Sci. 2015, 344, 27-32.

8. Wu, S.; Huang, H.; Feng, X.; Du, C.; Song, W. Facile Visual Colorimetric Sensor Based on Iron Carbide Nanoparticles Encapsulated in Porous Nitrogen-Rich Graphene. Talanta 2017, 167, 385-391.

9. Grass, R. N.; Athanassiou, E. K.; Stark, W. J. Covalently Functionalized Cobalt Nanoparticles as a Platform for Magnetic Separations in Organic Synthesis. Angew. Chem. Inter. Ed. 2007, 46, 4909-4912.

10. Zeng, M.; Liu, Y.; Zhao, F.; Nie, K.; Han, N.; Wang, X.; Huang, W.; Song, X.; Zhong, J.; Li, Y. Metallic Cobalt Nanoparticles Encapsulated in Nitrogen-Enriched Graphene Shells: Its Bifunctional Electrocatalysis and Application in Zinc-Air Batteries. Adv. Funct. Mater. 2016, 26, 4397-4404.

11. Hang, L.; Zhao, Y.; Zhang, H.; Liu, G.; Cai, W.; Li, Y.; Qu, L. Copper Nanoparticle@graphene Composite Arrays and Their Enhanced Catalytic Performance. Acta Mater. 2016, 105, 59-67.

12. Wang, Z.-J.; Wei, M.; Jin, L.; Ning, Y.; Yu, L.; Fu, Q.; Bao, X. Simultaneous NIntercalation and N-Doping of Epitaxial Graphene on 6h-SiC(0001) Through Thermal Reactions With Ammonia. Nano Res. 2013, 6, 399-408.

13. Hu, L.; Zhang, R.; Wei, L.; Zhang, F.; Chen, Q. Synthesis of FeCo Nanocrystals En- 
capsulated in Nitrogen-Doped Graphene Layers for Use as Highly Efficient Catalysts for Reduction Reactions. Nanoscale 2015, 7, 450-454.

14. Deng, J.; Ren, P.; Deng, D.; Bao, X. Enhanced Electron Penetration Through an Ultrathin Graphene Layer for Highly Efficient Catalysis of the Hydrogen Evolution Reaction. Angew. Chem. Int. Ed. 2015, 54, 2100-2104.

15. Ni, T.; Zhang, S.; Cao, F.; Ma, Y. NiCo Bimetallic Nanoparticles Encapsulated in Graphite-Like Carbon Layers as Efficient and Robust Hydrogenation Catalysts. Inorg. Chem. Front. 2017, 4, 2005-2011.

16. Luo, L.; Wu, J.; Luo, J.; Huang, J.; Dravid, V. P. Dynamics of Electrochemical Lithiation/Delithiation of Graphene-Encapsulated Silicon Nanoparticles Studied by In-situ TEM. Sci. Rep.. Sci. Rep. 2014, 4, 3863.

17. Kamali, A. R.; Kim, H.-K.; Kim, K.-B.; Vasant Kumar, R.; Fray, D. J. Large Scale Green Production of Ultra-High Capacity Anode Consisting of Graphene Encapsulated Silicon Nanoparticles. J. Mater. Chem. A 2017, 00, 1-10.

18. Zhang, M.; Liu, E.; Cao, T.; Wang, H.; Shi, C.; Li, J.; He, C.; He, F.; Ma, L.; Zhao, N. Sandwiched Graphene Inserted with Graphene-Encapsulated Yolk-Shell $\gamma-\mathrm{Fe}_{2} \mathrm{O}_{3}$ Nanoparticles for Efficient Lithium Ion Storage. J. Mater. Chem. A 2017, 5, 7035-7042.

19. Datteo, M.; Liu, H.; Di Valentin, C. Water on Graphene-Coated $\mathrm{TiO}_{2}$ : Role of Atomic Vacancies. ACS Appl. Mater. Interfaces 2018, 10, 5793-5804.

20. Zhang, X.; Xu, S.; Jiang, S.; Wang, J.; Wei, J.; Xu, S.; Gao, S.; Liu, H.; Qiu, H.; Li, Z.; Liu, H.; Li, Z.; Li, H. Growth Graphene on Silver-Copper Nanoparticles by Chemical Vapor Deposition for High-Performance Surface-Enhanced Raman Scattering. Appl. Surf. Sci. 2015, 353, 63-70. 
21. Ren, X.; Zi, W.; Wei, Q.; Liu, S. Fabrication Gallium/Graphene Core-Shell Nanoparticles by Pulsed Laser Deposition and Their Applications in Surface Enhanced Raman Scattering. Mater. Lett. 2015, 143, 194-196.

22. Tao, S.; Huang, W.; Xie, H.; Zhang, J.; Wang, Z.; Chu, W.; Qian, B.; Song, L. Formation of Graphene-Encapsulated $\mathrm{CoS}_{2}$ Hybrid Composites with Hierarchical Structures for High-Performance Lithium-Ion Batteries. RSC Adv. 2017, 7, 39427-39433.

23. Jiang, T.; Bu, F.; Liu, B.; Hao, G.; Xu, Y. Fe $7 \mathrm{SeSe}_{8} @ \mathrm{C}$ Core-Shell Nanoparticles Encapsulated within a Three-Dimensional Graphene Composite as a High-Performance Flexible Anode for Lithium-Ion Batteries. New J. Chem. 2017, 41, 5121-5124.

24. Myung, S.; Solanki, A.; Kim, C.; Park, J.; Kim, K. S.; Lee, K.-B. Graphene-Encapsulated Nanoparticle-Based Biosensor for the Selective Detection of Cancer Biomarkers. Adv. Mater. 2011 , 23, 2221-2225.

25. Wan, L. F.; Liu, Y. S.; Cho, E. S.; Forster, J. D.; Jeong, S.; Wang, H. T.; Urban, J. J.; Guo, J.; Prendergast, D. Atomically Thin Interfacial Suboxide Key to Hydrogen Storage Performance Enhancements of Magnesium Nanoparticles Encapsulated in Reduced Graphene Oxide. Nano Lett. 2017, 17, 5540-5545.

26. Cui, X.; Ren, P.; Deng, D.; Deng, J.; Bao, X. Single Layer Graphene Encapsulating Non-Precious Metals as High-Performance Electrocatalysts for Water Oxidation. Energy Environ. Sci. 2015, 9, 123-129.

27. Xu, Y.; Tu, W.; Zhang, B.; Yin, S.; Huang, Y.; Kraft, M.; Xu, R. Nickel Nanoparticles Encapsulated in Few-Layer Nitrogen-Doped Graphene Derived From Metal-Organic Frameworks as Efficient Bifunctional Electrocatalysts for Overall Water Splitting. Adv. Mater. 2017, 29, 1-8.

28. Deng, D.; Novoselov, K. S.; Fu, Q.; Zheng, N.; Tian, Z.; Bao, X. Catalysis With TwoDimensional Materials and Their Heterostructures. Nat. Nanotechnol. 2016, 11, 218-230. 
29. Fu, Q.; Bao, X. Surface Chemistry and Catalysis Confined Under Two-Dimensional Materials. Chem. Soc. Rev. 2017, 46, 1842-1874.

30. Martínez-Galera, A. J.; Schröder, U. A.; Huttmann, F.; Jolie, W.; Craes, F.; Busse, C.; Caciuc, V.; Atodiresei, N.; Blügel, S.; Michely, T. Oxygen Orders Differently Under Graphene: New Superstructures on Ir(111). Nanoscale 2016, 8, 1932-1943.

31. Conrad, H.; Ertl, G.; Latta, E. E. Adsorption of Hydrogen on Palladium Single Crystal Surfaces. Surf. Sci. 1974, 41, 435-446.

32. Penner, S.; Bera, P.; Pedersen, S.; Ngo, L. T.; Harris, J. J. W.; Campbell, Ch. T. Interactions of $\mathrm{O}_{2}$ with $\mathrm{Pd}$ Nanoparticles on $\alpha-\mathrm{Al}_{2} \mathrm{O}_{3}(0001)$ at Low and High $\mathrm{O}$ with $\mathrm{Pd}$ Nanoparticles on $\alpha-\mathrm{Al}_{2} \mathrm{O}_{3}(0001)$ at Low and High $\mathrm{O}_{2}$ Pressures. J. Phys. Chem. B 2006, $110,24577-24584$.

33. Banse, B. A.; Koel, B. E. Interaction of Oxygen with $\mathrm{Pd}(111)$ : High Effective $\mathrm{O}_{2}$ Pressure Conditions by Using Nitrogen Dioxide. Surf. Sci. 1990, 232, 275-285.

34. Surnev, L.; Bliznakov, G.; Kiskinova, M. Oxygen Adsorption on a Pd(111) Surface. Surf. Sci. 1984, 140, 249-260.

35. Ertl, G.; Koch, J. Adsorption von CO auf einer Palladium (111)-Oberfläche. Z. Naturforsch. A 1970, 25, 249-260.

36. Thiel, P. A.; Madey, T. E. The Interaction of Water With Solid Surfaces: Fundamental Aspects. Surf. Sci. Rep. 1987, 7, 211-385.

37. Heras, J. M.; Estiu, G.; Viscido, L. The Interaction of Water With Clean Palladium Films: A Thermal Desorption and Work Function Study. Appl. Surf. Sci. 1997, 108, 455-464. 
38. Barth, C.; Foster, A. S.; Henry, C. R.; Shluger, A. L. Recent Trends in Surface Characterization and Chemistry With High-Resolution Scanning Force Methods. Adv. Mater. 2011, 23, 477-501.

39. Glatzel, T.; Sadewasser, S.; Lux-Steiner, M. C. Amplitude or Frequency ModulationDetection in Kelvin Probe Force Microscopy. Appl. Surf. Sci. 2003, 210, 84-89.

40. Krok, F. Study of the Chemical and Morphological Evolution of InSb(001) Surface Under Low Energy Ion Bombardment. Vaccum 2009, 83, 745-751.

41. Palacios-Lidon, E.; Henry, C. R.; Barth, C. Kelvin Probe Force Microscopy in Surface Chemistry: Reactivity of Pd Nanoparticles on Highly Oriented Pirolytic Graphite. ACS Catal. 2014, 4, 1838-1844.

42. Ulbricht, H.; Zacharia, R.; Cindir, N.; Hertel, T. Thermal Desorption of Gases and Solvents From Graphite and Carbon Nanotube Surfaces. Carbon 2006, 44, 2931-2942.

43. Conrad, H.; Ertl, G.; Küppers, J.; Latta, E. E. Interaction of $\mathrm{NO}$ and $\mathrm{O}_{2}$ with $\mathrm{Pd}(111)$ surfaces. II. Surf. Sci. 1977, 65, 245-260.

44. Légaré, P.; Hilaire, L.; Maire, G.; Krill, G.; Amamou, A. Interaction of Oxygen and Hydrogen With Palladium. Surf. Sci. 1981, 107, 533-546.

45. Voogt, E. H.; Mens, A. J. M.; Gijzeman, O. L. J.; Geus, J. W. Adsorption of Oxygen and Surface Oxide Formation on $\operatorname{Pd}(111)$ and Pd Foil Studied With Ellipsometry, LEED, AES and XPS. Surf. Sci. 1997, 373, 210-220.

46. Zheng, G.; Altman, E. I. The Oxidation of Pd(111). Surf. Sci. 2000, 462, 151-168.

47. Leisenberger, F. P.; Koller, G.; Sock, M.; Surnev, S.; Ramsey, M. G.; Netzer, F. P.; Klötzer, B.; Hayek, K. Surface and Subsurface Oxygen on Pd(111). Surf. Sci. 2000, 445, 380-393. 
48. Körling, M.; Häglund, J. Cohesive and Electronic Properties of Transition Metals: The Generalized Gradient Approximation. Phys. Rev. B 1992, 45, 13293.

49. Todorova, M.; Reuter, K.; Scheffler, M. Oxygen Overlayers on Pd(111) Studied by Density Functional Theory. J. Phys. Chem. B 2004, 108, 14477-14483.

50. Todorova, M.; Reuter, K.; Scheffler, M. Density-Functional Theory Study of the Initial Oxygen Incorporation in Pd(111). Phys. Rev. B 2005, 71, 195403.

51. Romero-Muñiz, C.; Martín-Recio, A.; Pou, P.; Gómez-Rodríguez, J. M.; Pérez, R. Unveiling the Atomistic Mechanisms for Oxygen Intercalation in a Strongly Interacting Graphene-Metal Interface. Phys. Chem. Chem. Phys. 2018, 20, 13370-13378.

52. Zhang, H.; Fu, Q.; Cui, Y.; Tan, D.; Bao, X. Growth Mechanism of Graphene on $\mathrm{Ru}(0001)$ and $\mathrm{O}_{2}$ Adsorption on the Graphene/Ru(0001) Surface. J. Phys. Chem. C 2009, 113, 8296-8301.

53. Rose, M. K.; Borg, A.; Dunphy, J. C.; Mitsui, T.; Ogletree, D. F.; Salmeron, M. Chemisorption of Atomic Oxygen on Pd(111) Studied by STM. Surf. Sci. 2004, 561, 69-78.

54. Blanco, J. M.; González, C.; Jelínek, P.; Ortega, J.; Flores, F.; Pérez, R.; Rose, M.; Salmeron, M.; Méndez, J.; Wintterlin, J.; Ertl, G. Origin of Contrast in STM Images of Oxygen on Pd(111) and Its Dependence on Tip Structure and Tunneling Parameters. Phys. Rev. B 2005, 71, 113402.

55. Senftle, T. P.; Meyer, R. J.; Janik, M. J.; van Duin, A. C. T. Development of a ReaxFF Potential for Pd/O and Application to Palladium Oxide Formation. J. Chem. Phys. 2013, 139, 044109.

56. Nilsson, J.; Carlsson, P.-A.; Grönbeck, H.; Skoglundh, M. First Principles Calculations of Palladium Nanoparticle XANES Spectra. Top. Catal. 2017, 60, 283-288. 
57. Lundgren, E.; Kresse, G.; Klein, C.; Borg, M.; Andersen, J. N.; De Santis, M.; Gauthier, Y.; Konvicka, C.; Schmid, M.; Varga, P. Two-Dimensional Oxide on Pd(111). Phys. Rev. Lett. 2002, 88, 246103.

58. Klikovits, J.; Napetschnig, E.; Schmid, M.; Seriani, N.; Dubay, O.; Kresse, G.; Varga, P. Surface Oxides on Pd(111): STM and Density Functional Calculations. Phys. Rev. B 2007, 76, 045405.

59. Wrobel, R. J.; Becker, S.; Weiss, H. Influence of Subsurface Oxygen in the Catalytic CO Oxidation on $\operatorname{Pd}(111)$. J. Phys. Chem. C 2015, 119, 5386-5394.

60. Højrup Hansen, K.; Šljivancanin, Ž.; Lægsgaard, E.; Besenbacher, F.; Stensgaard, I. Adsorption of $\mathrm{O}_{2}$ and $\mathrm{NO}$ on Pd Nanocrystals Supported on $\mathrm{Al}_{2} \mathrm{O}_{3} / \mathrm{NiAl}(110)$ : Overlayer and Edge Structures. Surf. Sci. 2002, 505, 25-38.

61. Sadewasser, S.; Leendertz, C.; Streicher, F.; Lux-Steiner, M. Ch. The Influence of Surface Topography on Kelvin Probe Force Microscopy.. Nanotechnology 2009, 20, 505503.

62. Cohen, G.; Halpern, E.; Nanayakkara, S. U.; Luther, J. M.; Held, C.; Bennewitz, R.; Boag, A.; Rosenwaks, Y. Reconstruction of Surface Potential From Kelvin Probe Force Microscopy Images. Nanotechnology 2013, 24, 295702.

63. Barth, C.; Hynninen, T.; Bieletzki, M.; Henry, C. R.; Foster, A. S.; Esch, F.; Heiz, U. AFM Tip Characterization by Kelvin Probe Force Microscopy. New J. Phys. 2010, 12, 093024.

64. Hynninen, T.; Foster, A. S.; Barth, C. Polarized Tips or Surfaces: Consequences in Kelvin Probe Force Microscopy. e-J. Surf. Sci. Nanotech. 2011, 9, 6-14.

65. Ertl, G.; Kuppers, J. Interactions Between Oxygen and Carbon Monoxide on a Pd(111) Surface, Surf. Sci. 1978, 76, 323-342 
66. Mitsui, T.; Rose, M. K.; Fomin, E.; Ogletree, D. F.; Salmeron, M. Coadsorption and Interactions of $\mathrm{O}$ and $\mathrm{H}$ on $\mathrm{Pd}(111)$. Surf. Sci. 2002, 511, 259-266.

67. Petersson, L.-G.; Dannetun, H. M.; Lundström, I. The Water-Forming Reaction on Palladium. Surf. Sci. 1985, 161, 77-100.

68. Pauer, G.; Winkler, A. Water Formation on $\operatorname{Pd}(111)$ by Reaction of Oxygen with Atomic and Molecular Hydrogen, J. Chem. Phys. 2004, 120, 3864-3870.

69. Grånäs, E.; Knudsen, J.; Schröder, U. A.; Gerber, T.; Busse, C.; Arman, M. A.; Schulte, K.; Andersen, J. N.; Michely, T. Oxygen Intercalation Under Graphene on Ir(111): Energetics, Kinetics, and the Role of Graphene Edges. ACS Nano 2012, 6, 9951-9963.

70. Yao, Y.; Fu, Q.; Zhang, Y. Y.; Weng, X.; Li, H.; Chen, M.; Jin, L.; Dong, A.; Mu, R.; Jiang, P.; Liu, L.; Bluhm, H.; Liu, Z.; Zhang, S. B.; Bao, X. Graphene Cover-Promoted Metal-Catalyzed Reactions. Proc. Natl. Acad. Sci. 2014, 111, 17023-17028.

71. Sutter, P.; Sadowski, J. T.; Sutter, E. A. Chemistry Under Cover: Tuning MetalGraphene Interaction by Reactive Intercalation. J. Am. Chem. Soc. 2010, 132, 81758179.

72. Berry, V. Impermeability of Graphene and Its Applications. Carbon N. Y. 2013, 62, $1-10$.

73. Ni, S.; Li, Z.; Yang, J. Oxygen Molecule Dissociation on Carbon Nanostructures With Different Types of Nitrogen Doping. Nanoscale 2012, 4, 1184-1189.

74. Ma, L.; Zeng, X. C.; Wang, J. Oxygen Intercalation of Graphene on Transition Metal Substrate: An Edge-Limited Mechanism. J. Phys. Chem. Lett. 2015, 6, 4099-4105.

75. Palacio, I.; Otero-Irurueta, G.; Alonso, C.; Martínez, J. I.; López-Elvira, E.; MuñozOchando, I.; Salavagione, H. J.; López, M. F.; García-Hernández, M.; Méndez, J.; Ellis, G. J.; Martín-Gago, J. A. Chemistry Below Graphene: Decoupling Epitaxial Graphene 
From Metals by Potential-Controlled Electrochemical Oxidation. Carbon 2018, 129, 837846.

76. Barth, C.; Claeys, C.; Henry, C. R. Surface Preparation of Hard Ionic Crystals by Ultrahigh Vacuum Cleavage. Rev. Sci. Instr. 2005, 76, 083907.

77. Kitamura, S.; Suzuki, K.; Iwatsuki, M. High Resolution Imaging of Contact Potential Difference Using a Novel Ultrahigh Vacuum Non-Contact Atomic Force Microscope Technique. Appl. Surf. Sci. 1999, 140, 265-270.

78. We have used DMol, version 4.0

79. Delley, B. From Molecules to Solids With the DMol ${ }^{3}$ Approach. J. Chem. Phys. 2000, 113, 7756-7764.

80. Vosko, S. H.; Wilk, L.; Nusair, M. Accurate Spin-Dependent Electron Liquid Correlation Energies for Local Spin Density Calculations: A Critical Analysis. Can. J. Phys. 1980, 58,1200 .

81. Singh-Miller, N. E.; Marzari, N. Surface Energies, Work Functions, and Surface Relaxations of Low-Index Metallic Surfaces From First Principles. Phys. Rev. B. 2009, 80, 235407.

82. Patra, A.; Bates, J. E.; Sun, J.; Perdew, J. P. Properties of Real Metallic Surfaces: Effects of Density Functional Semilocality and van der Waals Nonlocality. Proc. Natl. Acad. Sci. 2017, 114, E9188-E9196. 


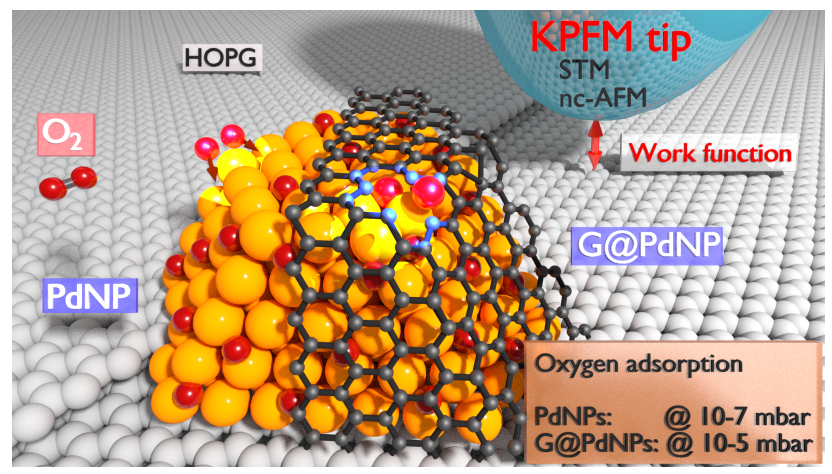

\section{TOC IMAGE}




\title{
- Supporting Information -
}

\section{Oxygen Adsorption on}

\section{Graphene Encapsulated Palladium Nanoparticles Imaged by Kelvin Probe Force Microscopy}

\author{
Henrik Grönbeck ${ }^{\dagger}$ and Clemens Barth ${ }^{*, \ddagger}$ \\ Chalmers University of Technology, Department of Physics and Competence Centre for Catalysis, SE-41296 \\ Göteborg, Sweden, and Aix-Marseille Université, CNRS, CINaM UMR 7325, 13288 Marseille, France \\ E-mail: barth@cinam.univ-mrs.fr
}

\section{Experimental methods}

Surface Preparation of HOPG. Fresh substrate surfaces are prepared by cleaving highly oriented pyrolytic graphite (HOPG) samples in air. Afterwards, the samples are quickly transfered into the ultra-high vacuum (UHV) chamber within a few minutes and the pumping as well as the bakeout of the UHV chamber $\left(\sim 100^{\circ} \mathrm{C}\right)$ are immediately started. Note that most of the surface contaminants, which adsorb from the air in the time between the cleavage and the transfer into the UHV chamber, are removed probably by the bakeout alone ${ }^{1}$. However, to guarantee utmost cleanness of the HOPG sample, the HOPG samples are cleaned by heating in an UHV oven ${ }^{2}$ kept at $\geq 650{ }^{\circ} \mathrm{C}$ during several hours. At the end of the annealing, with the sample still at $\sim 650^{\circ} \mathrm{C}$, the base pressure of the UHV is lower than $5 \times 10^{-10} \mathrm{mbar}$. This cleaning procedure is done a few times. As stated before ${ }^{3,4}$, such UHV annealed air-cleaved HOPG samples are as clean as UHV cleaved HOPG samples.

Growth of PdNPs. Palladium nanoparticles (PdNPs) are grown by evaporating palladium (Pd wire

\footnotetext{
* To whom correspondence should be addressed

${ }^{\dagger}$ Chalmers University of Technology, Department of Physics and Competence Centre for Catalysis, SE-41296 Göteborg, Sweden

${ }^{\ddagger}$ Aix-Marseille Université, CNRS, CINaM UMR 7325, 13288 Marseille, France
} 
with $99.95 \%$ purity, Goodfellow, Cambridgeshire, United Kingdom) at $\sim 1550{ }^{\circ} \mathrm{C}$ (measured with a $10 \% \mathrm{Rh}$ $\mathrm{Pt} / \mathrm{Pt}$ thermocouple) from a homemade Knudsen cell onto a HOPG sample, which is located in the UHV oven and kept between 470 and $500{ }^{\circ} \mathrm{C}$. Due to the Knudsen cell principle, only neutral palladium atoms are evaporated. With the calibrated deposition rate of $0.228 \mathrm{~nm} / \mathrm{min}(\sim 1$ mono-layer $(\mathrm{ML}) / \mathrm{min})$, a coverage with a nominal thickness of $1 \mathrm{ML}$ is obtained for a deposition time of 1 minute. During the growth, the base pressure never exceeds a total pressure of $3 \times 10^{-9}$ mbar. Immediately after the preparation of the NPs, the sample is extracted from the oven. At high temperatures (e.g., at $500{ }^{\circ} \mathrm{C}$ ), the temperature decreases by a few hundreds of degrees in the first 2 seconds and within a minute onto an estimated temperature of $<100^{\circ} \mathrm{C}$.

Growth of Graphene on PdNPs. With the UHV oven kept at a temperature between 670 and $740^{\circ} \mathrm{C}$, the PdNP/HOPG sample is placed inside the hot oven. After 7 minutes, the sample has reached the same temperature as the UHV oven. The UHV chamber is then back-filled with ethylene (99.8\%, Ethylene 2.8, Linde MINICAN, Munich, Germany) via a leakage valve while constantly monitoring the pressure. An ethylene pressure between 1.0 and $2 \times 10^{-6}$ mbar is used whereas the dosage is determined by the exposure time. The ion getter pump is switched off and only a turbo molecular pump is keeping the desired ethylene partial pressure. Typical exposure times are between 10 and 20 minutes. After the ethylene exposure, the sample is immediately extracted from the oven to avoid further reactions on the NPs at high temperatures. In the first 2 seconds the temperature decreases from, e.g., $670^{\circ} \mathrm{C}$ by some hundreds of degrees and within a minute onto an estimated temperature of $<200^{\circ} \mathrm{C}$.

Oxygen Dosage Experiments. For such experiments, only the UHV chamber with the AFM is used whereas the other parts of the UHV system are separated by a closed valve and remain at their best UHV pressure ( $\sim 10^{-10}$ mbar). The AFM/STM UHV chamber is back-filled with molecular oxygen (Oxygen 4.5, Linde MINICAN, Munich, Germany) via a leakage valve at a partial pressure between $3.0 \times 10^{-7}$ and $4.0 \times 10^{-5} \mathrm{mbar}$, whereas the dosage is determined by the exposure time. The ion getter pump is switched off and only a turbo molecular pump keeps the oxygen partial pressure at a desired value.

SPM System. Scanning probe microscopy (SPM) experiments are performed in an UHV chamber $\left(1 \times 10^{-10} \text { mbar base pressure }\right)^{2}$ with a room temperature (RT) Omicron AFM/STM (ScientaOmicron, Taunusstein, Germany). The AFM/STM is equipped with the SCALA electronics (ScientaOmicron, Taunusstein, Germany), which is controlled by the SCALA 4.1 and IDL 5.1 software running under SUN Solaris 2.5 on a SUN UNIX derivative. The graphical output of the SUN is sent to a Lubuntu ${ }^{5}$ GNU/Linux computer to increase the speed of the SCALA graphics ${ }^{6}$.

After the coarse approach of the surface to the tip, the surface is imaged for a relatively long time 
$(\geq 1 / 2$ hour) without recording any image. After this time most of the drift of the scanner is reduced and maximum stability is reached. Because of its relatively small influence with respect to an image frame of $\sim$ $500 \times 500 \mathrm{~nm}^{2}$, the residual lateral drift is only occasionally corrected by manually moving the image frame in the SCALA software.

All STM images are analyzed and prepared with the Gwyddion software ${ }^{7}$, whereas the color-maps from Matplotlib ${ }^{8}$ under Python ${ }^{9}$ are used for the 3D color space of the images.

STM. The scanning tunneling microscopy (STM) images are acquired in the constant current mode. The bias voltage is applied to the sample whereas the tip is at ground. STM experiments are conducted with electrochemically etched tungsten tips. Tungsten wires with a thickness of $0.38 \mathrm{~mm}$ are etched in a $\mathrm{NaOH}$ solution $(8.5 \mathrm{~g} \mathrm{NaOH}$ in $50 \mathrm{ml}$ water, $6.5 \mathrm{~mA}$ cutting current at $+4.0 \mathrm{~V})$.

nc-AFM. Noncontact atomic force microscopy (nc-AFM) is accomplished in the frequency modulation mode (self-excitation). Two conducting silicon cantilevers (PPP-QFMR, $\mathrm{n}^{+}-\mathrm{Si}, 0.01$ to $0.02 \Omega \mathrm{cm}$, NanoWorld AG, Neuchatel, Switzerland) with a resonance frequency of 71.4 (Figure 1 in the main article) and $78.7 \mathrm{kHz}$ (Figure 3 in the main article) are used, which amplitudes are kept constant during imaging. In the Omicron SCALA software, we use an amplitude of $0.1 \mathrm{~V}$, which corresponds to a rough guidance value of $25 \pm 10 \mathrm{~nm}$. The latter value is a mean value taken from 5 previously used tips of same type. To precisely measure the frequency shift $\triangle f$, a digital demodulator (EasyPLL, NanoSurf, Liestal, Switzerland) replaces the analogeous demodulator from the SCALA electronics.

KPFM. Kelvin probe force microscopy $(\mathrm{KPFM})$ is done in the frequency modulation mode ${ }^{10}$ where the dc bias voltage $\left(U_{\text {bias }}\right)$ and ac voltage $\left(U_{\text {ac }}\right.$, between 500 and $\left.650 \mathrm{mV}\right)$ with a frequency of $f_{\text {ac }}=630 \mathrm{~Hz}$ are applied at the sample (tip grounded). The electrostatic tip-surface interaction is minimized at each point on the surface by the bias voltage, which yields the contact potential difference (CPD) between tip and surface defined as $\mathrm{CPD}=U_{\text {bias }, 0}=\left(\phi_{\text {sample }}-\phi_{\text {tip }}\right) / e$ for the set-up used here. If at two different locations on the surface (Position 1 and 2) the CPD is measured, the CPD difference $\triangle \mathrm{CPD}=\mathrm{CPD}_{1}-\mathrm{CPD}_{2}=$ $\left(\left(\phi_{1}-\phi_{\text {tip }}\right)-\left(\phi_{2}-\phi_{\text {tip }}\right)\right) / e=\left(\phi_{1}-\phi_{2}\right) / e=\triangle \phi / e$ yields the work function difference $\triangle \phi_{1-2}=\phi_{1}-\phi_{2}$ between the two surface locations ( WF contrast) ${ }^{4,11}$. KPFM is applied during the constant frequency ncAFM imaging mode so that a topography and WF image of the CPD are simultaneously obtained. A bright and in particular orange/yellow contrast in WF images corresponds to a high WF whereas a violet and dark contrast corresponds to a low WF. Note that for a better data reading, the CPD values for HOPG are shifted onto zero such that the values of profiles are directly related to the WF difference between the PdNPs and HOPG (Figure 1 and 3 in the main article).

Analysis of Moiré Patterns. For all moiré patterns it is assumed that they have a perfect hexagonal 
structure. Furthermore, it is assumed that the edges of the NP's top (111) facet run along equivalent $<1 \overline{1} 0>_{\text {Pd }}$ surface directions ${ }^{12}$ and that the epitaxy between graphene and the (111) facets is commensurate as it is generally assumed for bulk $\operatorname{Pd}(111)$ surfaces $^{13,14}$. More details about the analysis can be found in the Supporting Information of Ref. [ 12] and [ 4].

\section{DFT CALCULATIONS}

The density functional theory (DFT) is used as implemented in the $\mathrm{Dmol}^{3}$ program ${ }^{15,16}$. The one-electron, Kohn-Sham orbitals are expanded using a local numerical basis set. The basis functions are atom-centered and stored on a radial grid ${ }^{15,16}$. A double numerical basis-set with polarization functions (dnp) is used for all atoms and a real space cut-off of $5 \AA$ is applied for all basis functions. A semi-core scalar relativistic pseudopotentials is used to describe the interactions between the valence electrons and the core for palladium ${ }^{17}$. Thus, 18 electrons are treated in the valence for palladium and 6 for carbon. The Kohn-Sham equations are solved self-consistently with an integration technique of weighted overlapping spheres located at each atomic center. The direct Coulomb potential is obtained by projection of the charge density onto angular dependent weighting functions also centered at each atom. The Poisson equation can in this way be solved by one-dimensional integration. The calculations are performed either within the local density approximation $(\mathrm{LDA})^{18}$ or with the generalized gradient approximation (GGA) using the functional proposed by Perdew, Burke, and Ernzerhof (PBE) ${ }^{19}$. LDA is used for evaluation of the work-fuction as this functional, thanks to error cancellation, is known to provide good results for surface properties ${ }^{20,21}$.

The $\operatorname{Pd}(111)$ surface is modeled with 7 layers using four different surface cells; $\sqrt{3} \times \sqrt{3}, 2 \sqrt{3} \times 2 \sqrt{3}$, $p(2 \times 2)$ and $p(5 \times 5)$. The slabs are separated by at least a $14 \AA$ vacuum including a dipole layer ${ }^{22}$. Integration over the Brillouin zone is approximated by finite sampling using a Monkhorst-Pack grid of $(8,8,1),(6,6,1)$, $(6,6,1)$ and $(3,3,1)$ for $\sqrt{3} \times \sqrt{3}, 2 \sqrt{3} \times 2 \sqrt{3}, p(2 \times 2)$ and $p(5 \times 5)$, respecively. Geometry optimization is performed using the BFGS method ${ }^{23-26}$ and the structures are regarded optimized when convergence criteria of $0.003 \mathrm{eV} / \AA, 0.0003 \mathrm{eV}$, and $0.003 \AA$ are meet for the largest gradient, total energy, and largest change in coordinates, respectively.

The work function $(\phi)$ is the energy needed to remove an electron from the bulk of palladium to the vacuum. It is calculated according to:

$$
\phi=V_{\text {vacuum }}-\epsilon_{F},
$$

where $V_{\text {vacuum }}$ is the electrostatic potential in the vacuum region and $\epsilon_{F}$ is the Fermi energy. 


\section{WF and Lattice Constant Values for Pd and HOPG}

We only consider experimental WF values, which were obtained exclusively in UHV, either by STM field emission using the Fowler-Nordheim analysis (STM_FE), target-current spectroscopy (TCS), two-photon photoemission spectroscopy (2PPS), time-resolved photoemission spectroscopy (TPS), ultraviolet photoelectron spectroscopy (UPS), angle resolved UPS (ARUPS) or metastable impact electron spectroscopy (MIES). The lattice constant of palladium was experimentally measured by X-ray scattering techniques (X-Ray) or by neutron scattering (NS). We compare values with the ones from theory, which were obtained by DFT, either computed with the local density approximation (LDA), generalized gradient approximation (GGA) or with other functionals.

HOPG. The WF values are listed in Table S1. A mean experimental literature value of $\phi_{\mathrm{HOPG}}$, lit,exp $=$ $4.53 \mathrm{eV}$ is found, which compares well with the theoretical value described in Ref. [ 27$]\left(\phi_{\mathrm{HOPG}}\right.$, theo $\left.=4.51 \mathrm{eV}\right)$. In our recent work ${ }^{4}$, we calibrated our HOPG surface against the $\operatorname{Ag}(001)$ surface and found a value of $\phi_{\mathrm{HOPG}}$, theo $=4.3 \pm 0.1 \mathrm{eV}$, which agrees with the literature value from above. For the entire work, we use our own calibrated value.

PdNPs. The WF of the Pd(111) surface can be used because all considered NPs made from palladium have a (111) top facet and a size still large enough to assume Pd bulk electronic properties. Table S2 shows that the experimental mean value of $\phi_{\operatorname{Pd}(111) \text {, lit, exp }}=5.64 \mathrm{eV}$ is in very good agreement with values from DFT theory. We also use a mean value for the lattice constant of palladium (Table S3). The experimental value $\left(a_{\mathrm{Pd}, \text { lit,exp }}=3.8898 \AA\right)$ agrees well with values from theory. 
Table S1: Experimental and DFT literature values for the work function $\phi(\mathrm{eV})$ of the HOPG surface. A mean value is listed for the experimental values $\left(\phi_{\mathrm{HOPG}, \mathrm{lit}, \exp }\right)$. Graphite here means natural graphite.

\begin{tabular}{llll}
\hline Surface & $\phi$ & Work & Reference \\
\hline Graphite & 4.6 & ARUPS & 28 \\
Graphite & 4.7 & TCS & 29 \\
HOPG & $4.5 \pm 0.1$ & 2 PPS & 3 \\
HOPG & 4.4 & UPS & 30 \\
HOPG & 4.4 & UPS & 31 \\
HOPG & 4.65 & UPS & 32 \\
HOPG & $4.50 \pm 0.05$ & TPS & 33 \\
HOPG & 4.6 & UPS & 34 \\
HOPG & 4.5 & STM_FE & 35 \\
HOPG & 4.45 & 2 PPS & 36 \\
\hline Exp. mean value & $4.53=\phi_{\text {HOPG,lit,exp }}$ & \\
\hline HOPG & 4.4 to 5.2 & LDA & 37 \\
HOPG & 4.51 & LDA & $27 *$ \\
\hline
\end{tabular}

Table S2: Experimental and DFT literature values for the work function $\phi(\mathrm{eV})$ of the $\mathrm{Pd}(111)$ surface. A mean value is listed for the experimental values $\left(\phi_{\operatorname{Pd}(111), \text { lit,exp }}\right)$.

\begin{tabular}{|c|c|c|c|}
\hline Surface & $\phi$ & Work & Reference \\
\hline $\operatorname{Pd}(111)$ & $5.6 \pm 0.2$ & UPS & 38 \\
\hline $\operatorname{Pd}(111)$ & $5.90 \pm 0.1$ & UPS & 39 \\
\hline $\operatorname{Pd}(111)$ & $5.95 \pm 0.1$ & UPS & 40 \\
\hline $\operatorname{Pd}(111)$ & 5.6 & UPS & 41 \\
\hline $\operatorname{Pd}(111)$ & 5.55 & MIES & 42 \\
\hline $\operatorname{Pd}(111)$ & $5.44 \pm 0.03$ & $2 \mathrm{PPS}$ & 43 \\
\hline $\operatorname{Pd}(111)$ & $5.50 \pm 0.01$ & $2 \mathrm{PPS}$ & 44 \\
\hline Exp. mean value: & $5.64=\phi_{\operatorname{Pd}(111), \text { lit }, \exp }$ & & \\
\hline $\operatorname{Pd}(111)$ & 5.53 & LDA & 45 \\
\hline $\operatorname{Pd}(111)$ & 5.9 & LDA & 46 \\
\hline $\operatorname{Pd}(111)$ & 5.75 & $\mathrm{LDA} \dagger$ & 47 \\
\hline $\operatorname{Pd}(111)$ & 5.42 & GGA (PW) & 48 \\
\hline $\operatorname{Pd}(111)$ & 5.74 & LDA & 49 \\
\hline $\operatorname{Pd}(111)$ & 5.86 & LDA & 50 \\
\hline $\operatorname{Pd}(111)$ & $5.64,5.22$ & LDA, GGA (PBE) & 51 \\
\hline $\operatorname{Pd}(111)$ & 5.67 & LDA & 52,53 \\
\hline $\operatorname{Pd}(111)$ & 5.59 & LDA & 14 \\
\hline $\operatorname{Pd}(111)$ & 5.39 & SCAN & 21 \\
\hline
\end{tabular}

*This work comments the theory work in Ref. 37 
Table S3: Experimental and DFT literature values for the lattice constant $a_{\mathrm{Pd}}$ of palladium $(\AA)$. A mean value is listed for the experimental values $\left(a_{\mathrm{Pd}, \text { lit,exp }}\right)$.

\begin{tabular}{llll}
\hline Material & $a$ & Work & Reference \\
\hline $\mathrm{Pd}$ & 3.958 & X-ray & 54 \\
$\mathrm{Pd}$ & 3.878 & X-ray & 55 \\
$\mathrm{Pd}$ & $3.859 \pm 0.003$ & X-ray & 56 \\
$\mathrm{Pd}$ & 3.881 & X-ray & 57 \\
$\mathrm{Pd}$ & 3.879 & X-ray & 58 \\
$\mathrm{Pd}$ & 3.8824 & X-ray & 59 \\
$\mathrm{Pd}$ & $3.889 \pm 0.001$ & X-ray & 60 \\
$\mathrm{Pd}$ & 3.889 & X-ray & 61 \\
$\mathrm{Pd}$ & 3.893 & X-ray & 62 \\
$\mathrm{Pd}$ & 3.89 & Neutron scattering & 63 \\
$\mathrm{Pd}$ & 3.8894 & Neutron scattering & 64 \\
\hline Exp. mean value: & $3.8898=a_{\mathrm{Pd}, \mathrm{lit}, \text { exp }}$ & & 65 \\
\hline $\mathrm{Pd}$ & 3.96 & GGA $($ PW91) \\
$\mathrm{Pd}$ & 3.85 & LDA & 51 \\
$\mathrm{Pd}$ & 3.95 & GGA & 66 \\
$\mathrm{Pd}$ & 3.889 & LDA & 67 \\
$\mathrm{Pd}$ & 3.88 & See note $\S$ & 68 \\
$\mathrm{Pd}$ & 3.87 & See note $\mathbb{I}$ & 69 \\
$\mathrm{Pd}$ & 3.90 & See note $\|$ & 70 \\
$\mathrm{Pd}$ & 3.954 & GGA & 21 \\
\hline
\end{tabular}

\section{Supporting experiments}

The WF of the AFM Tip During $\mathrm{O}_{2}$ Exposure. Figure S1 shows the same KPFM images of the as-prepared PdNPs, which can be seen in Figure 1 of the main article. Apart from the topography images in the top row, which are identical, the WF images show raw values unlike in Figure 1 of the main article. Note that the absolute contrast of a WF image (a specific colour) is directly related to the CPD between the surface and tip: $\mathrm{CPD}=\left(\phi_{\text {sample }}-\phi_{\text {tip }}\right) / e$.

Before the oxygen dosage starts (Figure S1a and b), the NPs are imaged in UHV (blue arrows). The WF

†With Ceperley-Alder exchange-correlation potential

$\ddagger$ Several different functionals. We only consider the SCAN value.

§Several different functionals (LDA, PBE, PBEsol, etc.). We only consider the four values in bold of table I.

ISeveral different GGA functionals. We calculated the averaged value from the three values in bold in Table II.

$\|$ Several different functionals (LDA, PBE, etc.). An average value is used from RPA and RPA + .

**Several different functionals. We only consider the SCAN value. 

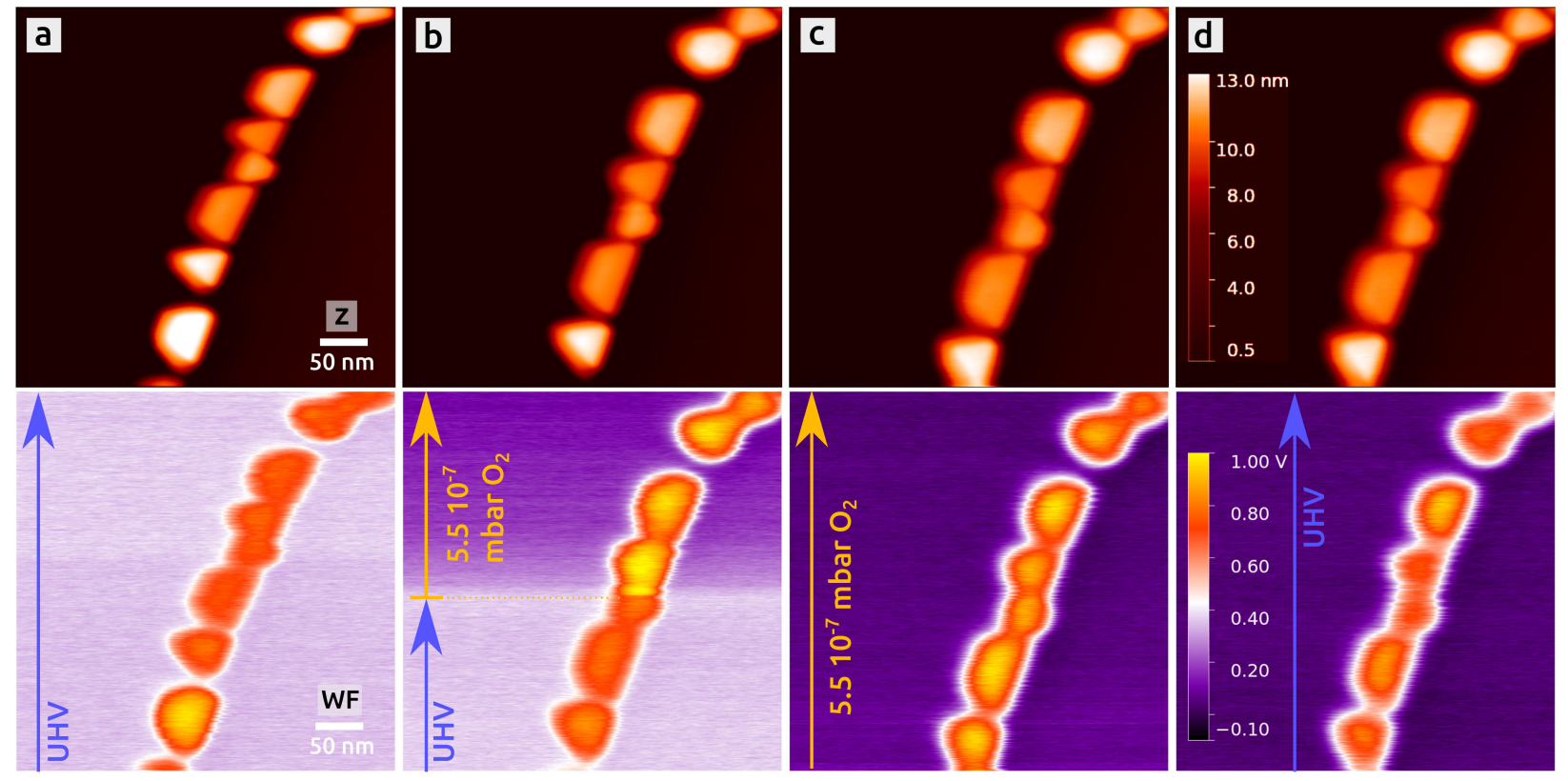

Figure S1: As-prepared PdNPs imaged by KPFM during an exposure of $\mathrm{O}_{2}$ at room temperature $\left(p_{\mathrm{O} 2}=3 \times 10^{-7} \mathrm{mbar}\right.$, exposure time $\left.57 \mathrm{~min} \equiv 771 \mathrm{~L}\right)$. The WF images (bottom row) show the raw data from the images in Figure 1 of the main article whereas the topography images (top row) are the same as in Figure 1. One KPFM experiment is represented by a pair of two vertically arranged images. The orange arrows show the $\mathrm{O}_{2}$ exposure time whereas blue arrows correspond to the imaging in UHV (no $\mathrm{O}_{2}$ ). NP growth: $2.0 \mathrm{ML}$ at $476{ }^{\circ} \mathrm{C}$, KPFM parameters: $\triangle f=-8.2 \mathrm{~Hz}, v=0.5 \mathrm{~Hz}, U_{\mathrm{ac}}=350 \mathrm{mV}$ and $f_{\mathrm{ac}}=650 \mathrm{~Hz}$.

of the NPs and the HOPG surface do not change, as well as not the WF of the tip. However, from the start of the oxygen dosage in Figure S1b (yellow arrow), the WF of the NPs seems to increase (from dark orange to bright orrange) whereas the WF of HOPG seems to decrease (from white to dark violet). The temporal development of the CPD above the HOPG surface can be also seen by the CPD vs time curve in Figure S2a.

Whereas the NPs do indeed change their WF (see below), the apparent WF decrease on the HOPG surface is not due an adsorption of oxygen on HOPG because the terraces of the surface are inert towards any adsorption of gas molecule at $\mathrm{RT}^{80}$. It is rather the WF of the tip, which changes during the oxygen dosage experiment: the AFM tip is made from crystalline silicon, which is conducting due to the $\mathrm{n}^{+}$implantation that was done during the industrial production of the tip. The tip apex carries a some nanometer thick native oxide since the tip is exposed to the ambient air before it is transferred into the UHV chamber. During the nc-AFM imaging of a PdNP/HOPG sample surface, the tip is sometimes in contact with the sample (tip-change), in particular with the NPs, due to instabilities in the tip-surface interaction, which regularly appear from time to time and which cannot be avoided. Therefore, the tip apex may be composed by either some silicon oxide or even silicon at places at the tip apex, where the oxide has been broken during a tip-change. The tip apex might be also contaminated by some palladium, which originates from the NPs. When such a tip is exposed to oxygen, the tip apex certainly oxidizes. When, e.g., silicon and/or palladium 

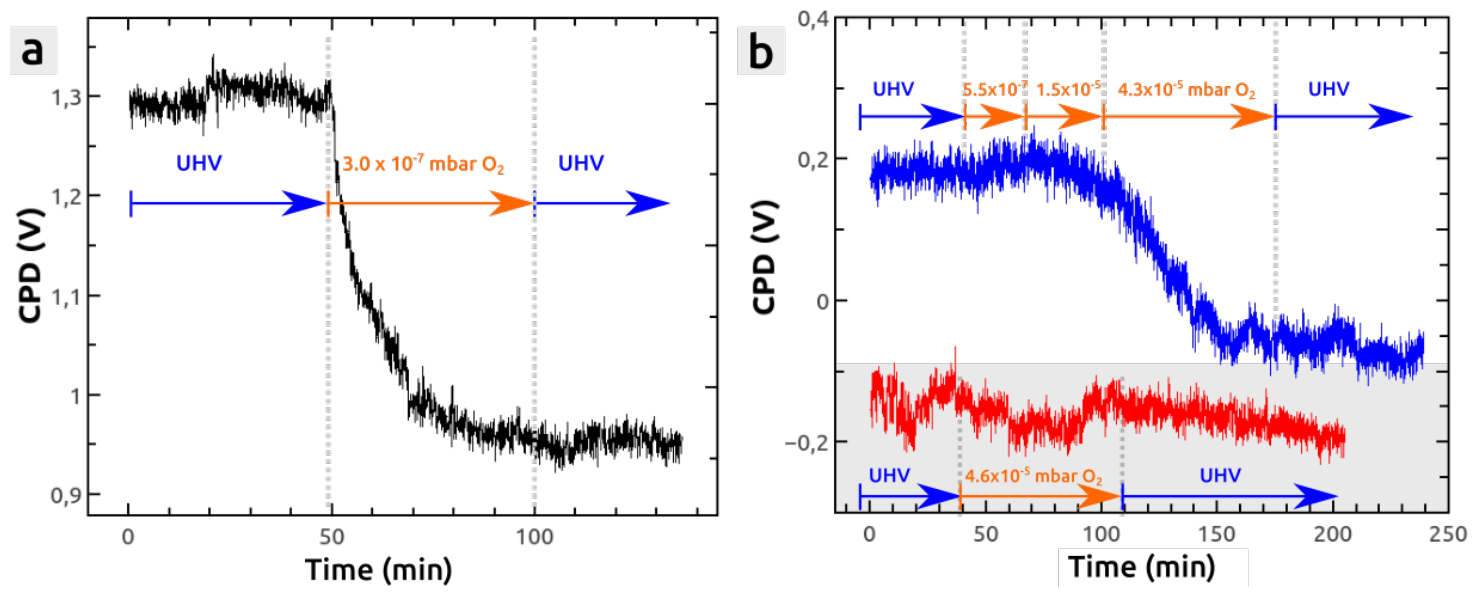

Figure S2: The temporal development of the CPD measured between the HOPG surface and the AFM tip upon the $\mathrm{O}_{2}$ exposure shown in Figure S1, Figure S3 and Figure S4, displayed by the black curve (a), and the blue and red curve (b), respectively. The CPD vs time values were extracted from the raw WF images shown in the latter three Figures. More details can be found in the text.

get oxidized at RT they increase their WF (see Table S4), which is evident for Pd as shown in this work. Because the $\mathrm{CPD}$ is proportional to $-\phi_{\text {tip }}\left(\mathrm{CPD}=\left(\phi_{\text {sample }}-\phi_{\text {tip }}\right) / e\right)$, the $\mathrm{CPD}$ decreases what can be seen in Figure S2a and b (blue curve). However, if the tip is already oxidized, there are no visible changes in the $\mathrm{CPD}$, as it will be discussed further below with Figure S4 and Figure S2b (red curve).

Because the WF of the HOPG terraces does not change and because of the tip's WF decrease in oxygen, a line leveling can be done as follows: with the 'Path Leveling Tool' in the Gwyddion software ${ }^{7}$, only straight lines on the HOPG terraces are selected in the entire WF image. The WF on the HOPG terraces is then leveled, removing the WF decrease. The result is that all places on the HOPG terraces are on the same WF (same colour) and that the absolute CPD values at the NPs are corrected, from the WF increase of the tip. Overall, this procedure yields the correct WF difference between the NPs and the HOPG surface, as it can be seen in Figure 1 of the main article. Note that for a better data reading, the CPD values for HOPG are shifted onto zero such that the values of the profiles (Figure 1 of the main article) are directly related to the WF difference between the PdNPs and HOPG.

The same leveling procedure is done for the WF data in Figure 3 of the main article. Figure S3 shows the raw data of the WF images (middle row) where a similar increase of the tip's WF upon oxygen exposure can be seen (see blue curve in FigureS2b).

Figure $\mathrm{S} 4$ shows the same G@PdNPs during a second oxygen dosage experiment under similar conditions. Because the tip has been already fully oxidized in the first dosage experiment (Figure S3), no dramatic WF changes can be seen in the raw WF images in Figure S4 (middle row). The red CPD vs time curve in Figure S2b shows only slight WF changes in the order of a few tens of $\mathrm{mV}$. 
Table S4: Work function $(\phi)$ values in $\mathrm{eV}$ for silicon and palladium as well as for adsorbed oxygen. If applicable, the WF difference between the clean material and adsorbed oxygen $(\triangle \phi)$ is listed.

\begin{tabular}{|c|c|c|c|c|}
\hline Material & $\phi$ & $\triangle \phi$ & Notes & Reference \\
\hline Si, polycristalline & 4.85 & / & Exp. & 71 \\
\hline \multirow[t]{2}{*}{$\operatorname{Si}(100)$} & 4.92 & / & Exp. & 72 \\
\hline & $4.88-4.97$ & / & DFT & 73 \\
\hline $\operatorname{Si}(110)$ & 4.89 & / & Exp. & 72 \\
\hline \multirow[t]{2}{*}{$\operatorname{Si}(111)$} & 4.77 & / & Exp. & 72 \\
\hline & $4.64-4.96$ & / & $\mathrm{DFT}$ & 73 \\
\hline $\mathrm{Si}(111), \mathrm{p}$ to $\mathrm{n}$ doping & 4.7 to 4.9 & / & Exp., max. doping levels for $\mathrm{n}$ and $\mathrm{p}$ doped samples & 74 \\
\hline O on $\mathrm{Si}(100)$ and $\mathrm{Si}(110)$ & $\sim 5.2$ & $+0.1 \ldots+0.2$ & Exp., $\mathrm{O}_{2}$ dosage at $\mathrm{RT}$ until saturation & 72 \\
\hline O on $\mathrm{Si}(111)$ & $\sim 5.2$ & $+0.3 \ldots+0.4$ & Exp., $\mathrm{O}_{2}$ dosage at $\mathrm{RT}$ until saturation & 72 \\
\hline O on $\mathrm{Si}(111) 7 \times 7$ & & $+0.9(+0.2)$ & Exp., after a few $\mathrm{L}(>50 \mathrm{~L})$ & 75,76 \\
\hline O on $\mathrm{Si}(100)$ & & $+0.2 \ldots+1.0$ & Exp., thermal oxidation at high Ts $\left(800-1000^{\circ} \mathrm{C}\right)$ & 77 \\
\hline $\mathrm{Pd}$, polycristalline & 5.1 & / & Exp. & 71 \\
\hline $\operatorname{Pd}(111)$ & 5.7 & / & Exp. & See Table S2 \\
\hline $\operatorname{Pd}(100)$ & 5.5 & / & Exp. & 78,21 \\
\hline $\operatorname{Pd}(110)$ & 5.1 & / & Exp. & 78,21 \\
\hline O on $\mathrm{Pd}(111)$ & $\geq 6.2$ & $\geq+0.5$ & Exp., oxygen adsorbed on $\operatorname{Pd}(111)$ at $\mathrm{RT}$ & This work \\
\hline $\mathrm{O}$ on $\mathrm{Pd}(110)$ & $\sim 5.5$ & $\sim+0.4$ & Exp., oxygen adsorbed on $\mathrm{Pd}(111)$ at around $\mathrm{RT}$ & 79 \\
\hline
\end{tabular}
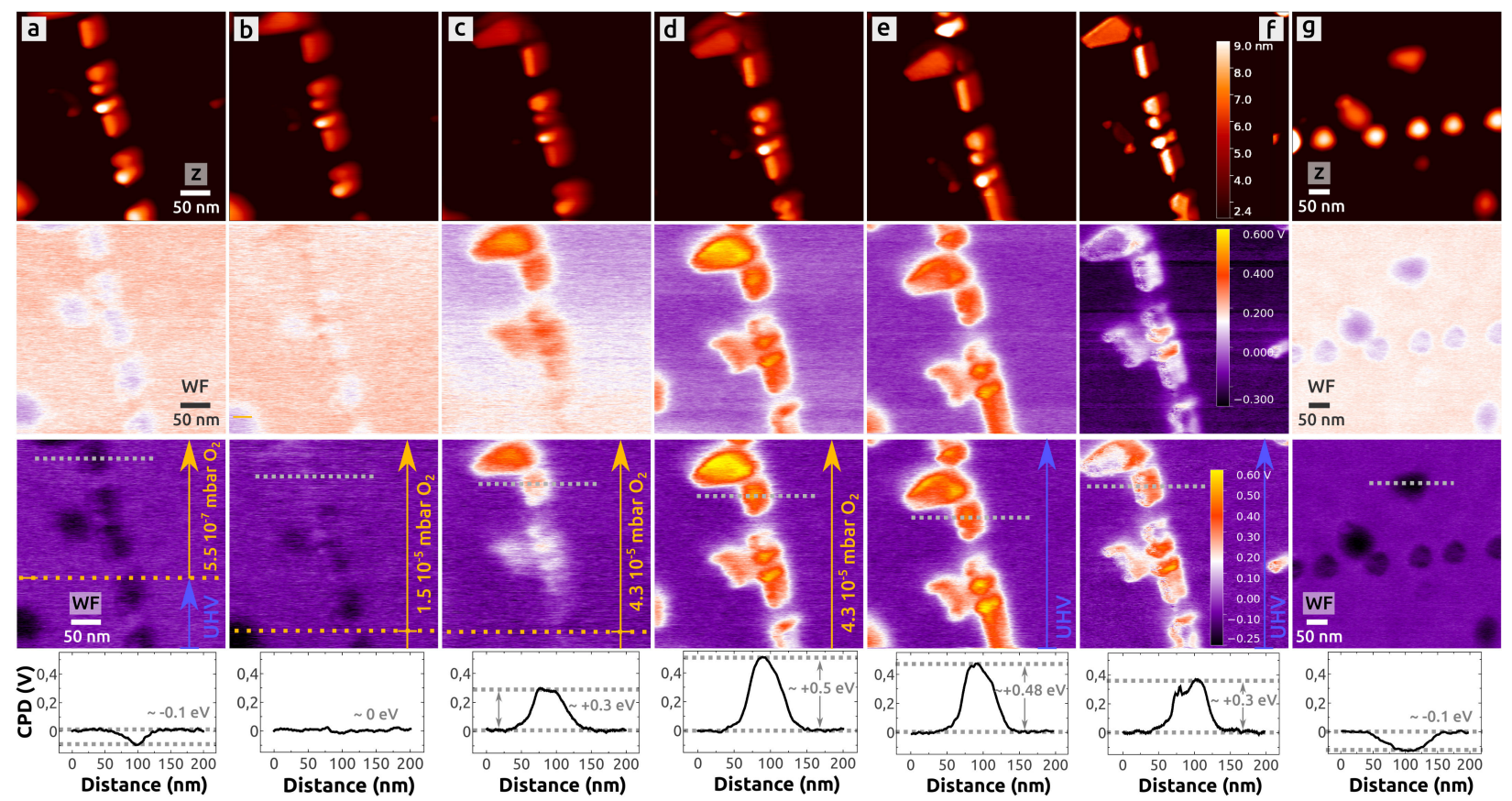

Figure S3: Graphene encapsulated PdNPs imaged by KPFM during an exposure of $\mathrm{O}_{2}$ at room temperature $\left(620 \mathrm{~L} @ p_{\mathrm{O} 2}=5.5 \times 10^{-7} \mathrm{mbar}(\mathrm{a}), 2.2 \times 10^{4} \mathrm{~L} @ 1.5 \times 10^{-5} \mathrm{mbar}(\mathrm{b})\right.$ and $1.5 \times 10^{5} \mathrm{~L} @ 4.3 \times 10^{-5} \mathrm{mbar}(\mathrm{c})$, total dosage: $1.7 \times 10^{5} \mathrm{~L}$ ). One KPFM experiment is represented by a pair of three vertically arranged images with a representative WF profile underneath. The topography images (top row) are the same as in Figure 3 of the main article. The WF images in the middle show the raw data whereas the WF images at the bottom are corrected from changes of the tip's WF. The orange arrows show the $\mathrm{O}_{2}$ exposure whereas blue arrows correspond to the imaging in UHV $\left(\right.$ no $\left.\mathrm{O}_{2}\right)$. NP growth: $1.5 \mathrm{ML}$ at $475{ }^{\circ} \mathrm{C}$, Graphene growth: $847 \mathrm{~L}$ of $\mathrm{C}_{2} \mathrm{H}_{4}\left(1 \times 10^{-6}\right.$ mbar $)$ at $670^{\circ} \mathrm{C}, \mathrm{KPFM}$ parameters: $\triangle f=-9.5$ (a,b), $-10.55(\mathrm{c}),-12.65$ (d), -13.61 (e,f) and $-15.3 \mathrm{~Hz}(\mathrm{~g})$, all: $v=0.5 \mathrm{~Hz}, U_{\mathrm{ac}}=500 \mathrm{mV}$ and $f_{\mathrm{ac}}=630 \mathrm{~Hz}$. 

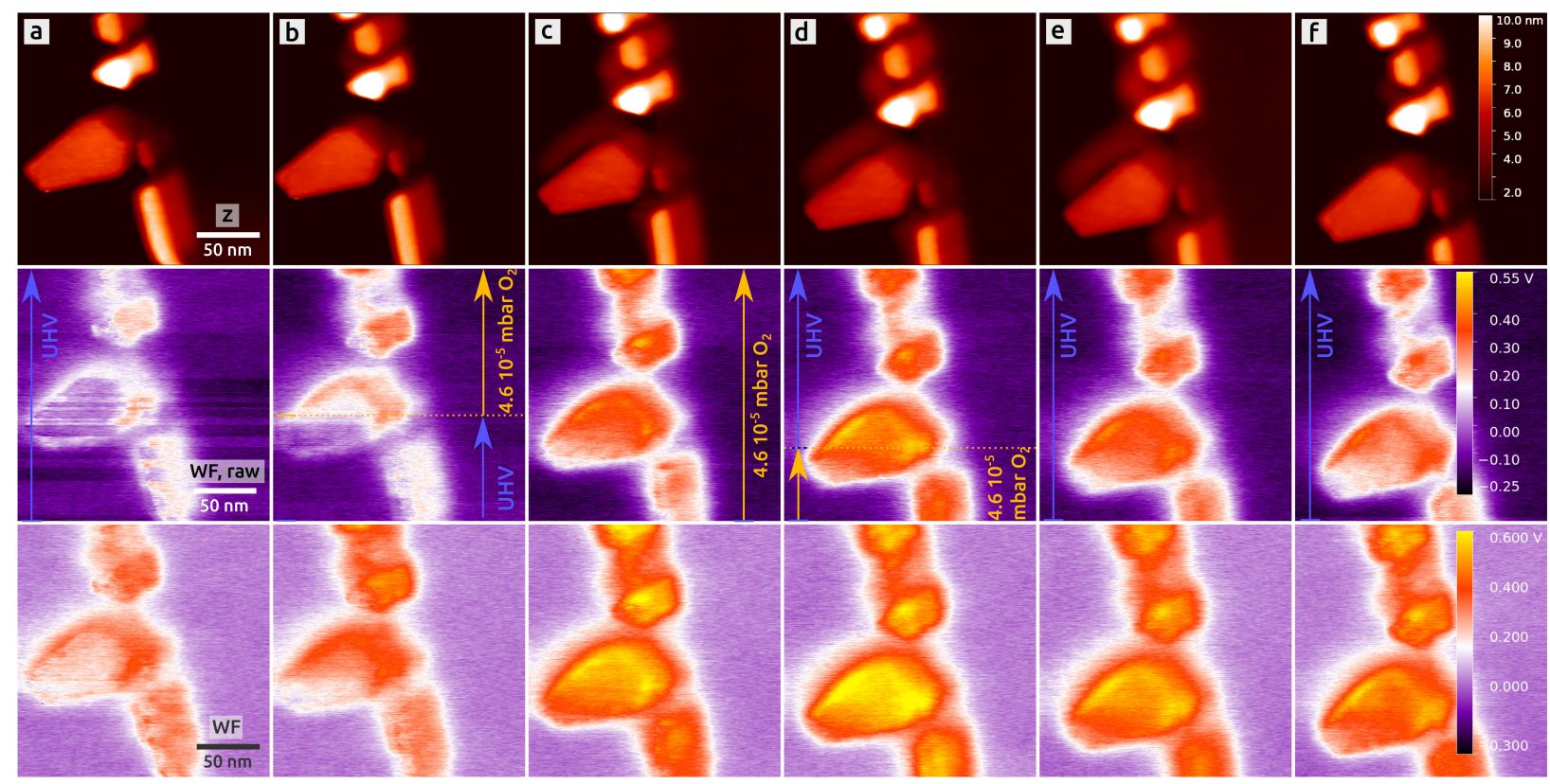

Figure S4: Same sample as in Figure S3 with G@PdNPs imaged by KPFM during a second exposure of $\mathrm{O}_{2}$ at room temperature $\left(1.4 \times 10^{5} \mathrm{~L} @ p_{\mathrm{O} 2}=4.6 \times 10^{-5} \mathrm{mbar}\right.$, exposure time $\left.66 \mathrm{~min}\right)$. One KPFM experiment is represented by a pair of three vertically arranged images. The images on the top represent the topography (z) whereas the images in the second and third row represent the raw (second row) and leveled WF data (third row). The orange arrows show the $\mathrm{O}_{2}$ exposure whereas blue arrows correspond to the imaging in UHV (no $\mathrm{O}_{2}$ ). NP and graphene growth: see Figure S3, KPFM parameters: $\triangle f=-10.2$ (a-e) and $-12.3 \mathrm{~Hz}$ (f), all: $v=0.5 \mathrm{~Hz}, U_{\mathrm{ac}}=500 \mathrm{mV}$ and $f_{\mathrm{ac}}=630 \mathrm{~Hz}$.

\section{SUPPORTING THEORY}

Model of Dissociation/Adsorption of Oxygen. In the main article it is shown that the dissociative adsorption of oxygen on as-prepared PdNPs almost completely saturates within a few seconds at a partial oxygen pressure of $p_{\mathrm{O} 2 \text {, PdNPs }}=3 \times 10^{-7}$ mbar. In comparison, an almost two order of magnitude higher partial pressure is needed for observing on the same time scale a similar oxygen adsorption on G@PdNPs $\left(p_{\mathrm{O} 2, \mathrm{G} @ \mathrm{PdNPs}} \approx 4 \times 10^{-5} \mathrm{mbar}\right)$. The reason for this large difference is that graphene covers almost all palladium atoms where dissociation and adsorption of oxygen normally occurs on as-prepared NPs. It is clear that oxygen can only dissociate at palladium atoms below the graphene defects so that atomic oxygen can then diffuse inside the nanocontainer. Here we show, that the two order of magnitude higher pressure can be explained if it is assumed that most of the graphene defects are, e.g., at the NP's edges.

We consider the NP's top (111) facet, which is formed by two types of palladium atoms: (a) atoms on the facets and (b) atoms forming the edges of the facets. The ratio of the atom numbers of both atom types, $N_{\text {Facet }}$ and $N_{\text {Edge }}$, can then be assumed, in a first rough approximation, to be proportional to the ratio of partial oxygen pressures observed at as-prepared PdNPs and G@PdNPs: 


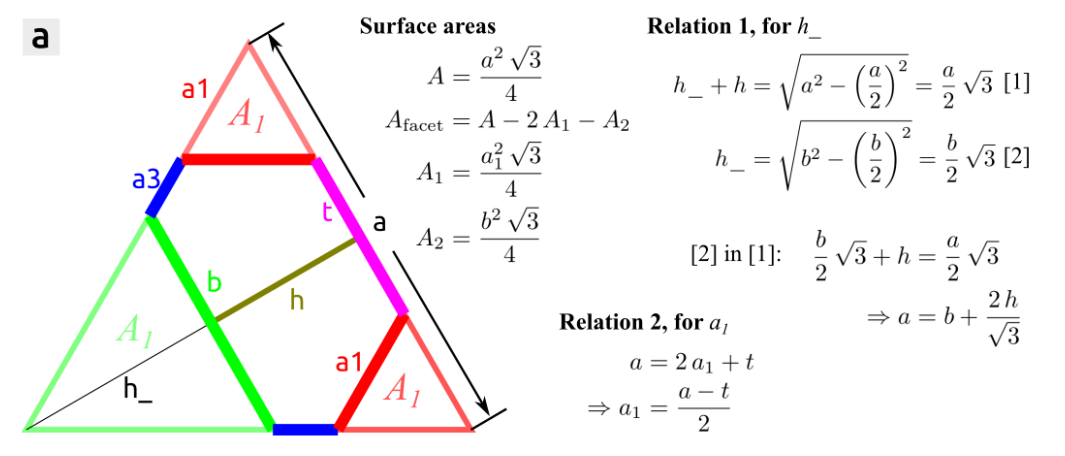

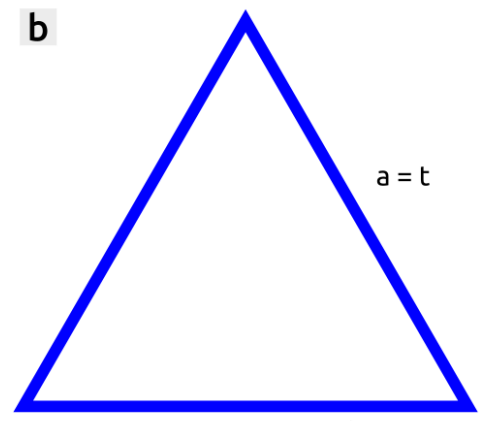

$\mathrm{a}=\mathrm{t}, \mathrm{b}=0, \mathrm{~h}=\mathrm{t} \sqrt{ } 3 / 2$

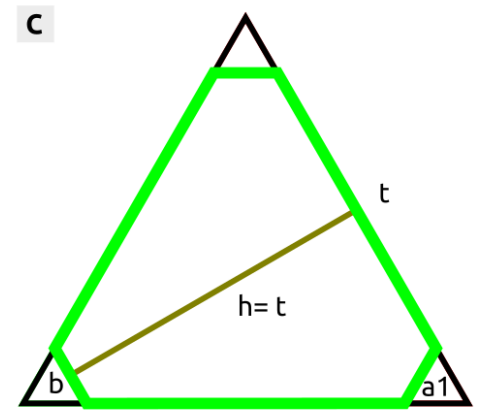

$\mathrm{h}=\mathrm{t}, \mathrm{b}=\mathrm{a}_{1}=2 \mathrm{t} / \sqrt{3}-\mathrm{t}$

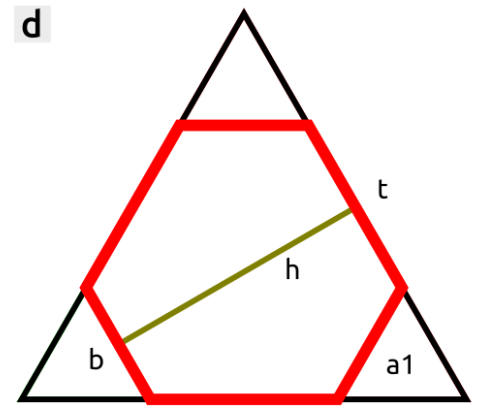

$\mathrm{a}_{1}=\mathrm{b}=2 / 3 \mathrm{t}$

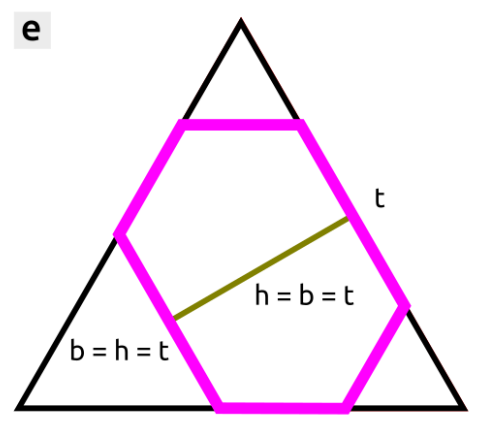

$\mathrm{b}=\mathrm{h}=\mathrm{t}$

Figure S5: The shape of the top (111) facet of a PdNP is a equilateral triangle (tetarhedron), which is mostly truncated. (a) The truncation of the triangle with equilateral sub-triangles used for the calculation of the surface area. (b-e) Frequently observed shapes of the top facet (blue, green, red and pink outline): triangle (b), corner truncated triangle (c), hexagon with edges of comparable length (d) and assymetric long-shaped hexagon (e).

$$
r=\frac{N_{\text {Facet }}}{N_{\text {Edge }}} \sim \frac{p_{\mathrm{O} 2, \mathrm{PdNPs}}}{p_{\mathrm{O} 2, \mathrm{G} @ \text { PdNPs }}} \approx 100
$$

The atom numbers can be calculated from the densities of atoms on the facet $\left(\varrho_{\text {Facet }}\right.$, in atoms $\left./ \mathrm{cm}^{2}\right)$ and at the edges $\left(\varrho_{\text {Edge }}\right.$, in atoms $\left./ \mathrm{cm}\right)$, and from the surface area of the facet $\left(A_{\text {Facet }}\right)$ and total length of all edges $\left(L_{\text {Edge }}\right)$.

$$
\begin{aligned}
& N_{\text {Facet }}=A_{\text {Facet }} \varrho_{\text {Facet }} \\
& N_{\text {Edge }}=L_{\text {Edge }} \varrho_{\text {Edge }}
\end{aligned}
$$

We consider real shapes of top (111) facets, which can be frequently observed at PdNPs. Due to their $3 \mathrm{D}$ shape of a top-truncated tetrahedron and their $(111)_{\mathrm{Pd}} \|(00.1)_{\mathrm{HOPG}}$ and $[11 \overline{2}]_{\mathrm{Pd}} \|[1 \overline{1} .0]_{\mathrm{HOPG}}$ epitaxial orientation on HOPG, the facets exhibit shapes from triangles to hexagons via various truncated shapes, with the NPs' edges forming always angles of $60^{\circ}$ and $120^{\circ}$. Some selected, frequently observed shapes are 
shown in Figure S5b-e (see thick outline in blue (b), green (c), red (d) and pink (e)).

The surface area and total edge length can be calculated by considering three parameters, e.g., the height $h$, and the bottom $b$ and top base length $t$ (Figure S5a). From geometric considerations, which are summarized in Figure S5a, the surface area and length can be obtained by:

$$
\begin{aligned}
A_{\text {Facet }} & =\frac{\sqrt{3}}{4}\left(a^{2}-\frac{(a-t)^{2}}{2}-b^{2}\right) \\
\text { with } a & =b+\frac{2 h}{\sqrt{3}} \\
L_{\text {Edge }} & =t+b+\frac{4 h}{\sqrt{3}}
\end{aligned}
$$

The four model shapes from Figure S5b-e are used to calculate the ratio $r$, which the latter can be obtained by inserting Eq. (4) and (6) into Eq. (2) and (3), respectively. In the following, we consider the following conditions for the four shapes in FigureS5:

- b: $a=t, b=0$ and $h=\frac{\sqrt{3}}{2} t$

$$
\begin{aligned}
& A_{\text {Facet }}=\frac{\sqrt{3}}{4} t^{2} \\
& L_{\text {Edge }}=3 t
\end{aligned}
$$

- c: $b=a_{1}=\left(\frac{2}{\sqrt{3}}-1\right) t$ and $h=t$

$$
\begin{aligned}
& A_{\text {Facet }}=\frac{\sqrt{3}}{6}(2 \sqrt{3}-1) t^{2} \\
& L_{\text {Edge }}=2 \sqrt{3} t
\end{aligned}
$$

- d: $b=\frac{2}{3} t$ and $h=\frac{5}{2 \sqrt{3}} t$

$$
\begin{aligned}
A_{\text {Facet }} & =\frac{37}{36} \sqrt{3} t^{2} \\
L_{\text {Edge }} & =5 t
\end{aligned}
$$

- e: $b=h=t$

$$
A_{\text {Facet }}=\left(1+\frac{\sqrt{3}}{6}\right) t^{2}
$$




$$
L_{\text {Edge }}=2\left(1+\frac{2}{\sqrt{3}}\right) t
$$

The parameters $b$ and $h$ are now a function of $t$, which the latter shall be a measure of the facet's size. With this, we obtain four equations in the form $A_{\text {Facet }}=A_{\text {Facet }}\left(t^{2}\right)$ and four equations in the form $L_{\text {Edge }}=$ $L_{\mathrm{Edge}}(t)$. The related ratios $r$ are then a linear function of $t$. Note that we have to normalize the lateral size of the facets: the radius of a circle is calculated from the surface area $A_{\text {Facet, } \triangle}$ of the triangular facet (Figure S5b) and is devided by the radius of a circle with surface area $A_{\text {Facet }}$ of a considered facet (Figure S5ce). The latter ratio is multiplied with the size $t$ of the respective facet as follows:

$$
t_{\text {Norm }}=t \times\left(\sqrt{\frac{A_{\text {Facet }, \triangle}}{\pi}} / \sqrt{\frac{A_{\text {Facet }}}{\pi}}\right)
$$

For the densities, $\varrho_{\text {Facet }}$ and $\varrho_{\text {Edge }}$, we assume that all palladium atoms on the facet and at the edges, respectively, are active sites for the dissociation. With the next-neighbour $\mathrm{Pd}-\mathrm{Pd}$ distance $e_{\mathrm{Pd}}=a_{\mathrm{Pd}} / \sqrt{2}$, we have the following densities:

$$
\begin{aligned}
\varrho_{\text {Facet }} & =\frac{1}{e_{\mathrm{Pd}}^{2} \sin \left(60^{\circ}\right)}=\frac{2}{e_{\mathrm{Pd}}^{2} \sqrt{3}} \\
\varrho_{\text {Edge }} & =\frac{1}{2} \times \frac{1}{e_{\mathrm{Pd}}}
\end{aligned}
$$

Note that we have to use a factor of $\frac{1}{2}$ for the edge density because two facets form one edge, and each facet has a $50 \%$ probablity to receive the oxygen adsorbates.

The four curves in the lower part of Figure S6 show the ratio $r=N_{\text {Facet }} / N_{\text {Edge }}$ versus the normalized lateral size of the facet, $\mathrm{t}_{\text {Norm }}$, for the four typical facets shown in Figure S5b-e. The ratio is a linear function of the size $\left(t_{\text {Norm }}\right)$ for all facets, whereas only small differences can be seen. The triangular shape has the smallest slope because there are relative more edge sites with respect to the number of edges of the other shapes, which maximize the surface area with respect to their total edge length. A ratio of around 100 can be found for lateral NP sizes between 70 and $80 \mathrm{~nm}$.

The NP's sizes, which can be seen in the images of Figure 3 (main article) or Figure S3, ranges between 10 and $50 \mathrm{~nm}$. With this the calculated ratio $r$ yields a value between 20 and 60 , which is too small. It seems that not all edge sites are active sites for the dissociation. If we now assume that only each second site at the edges is an active site ( 4 curves in the upper part of Figure S6), we obtain a ratio of 100 for NP sizes of around $35 \mathrm{~nm}$, which falls into the range of NP sizes observed in our experiments. 


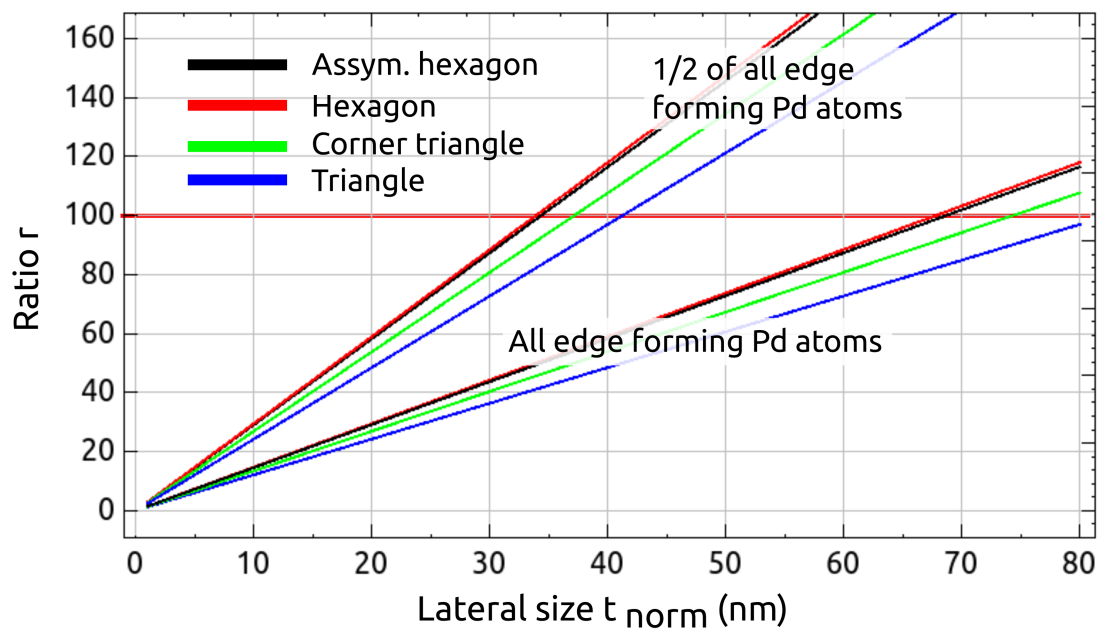

Figure S6: The ratio $r=N_{\text {Facet }} / N_{\text {Edge }}$ versus the normalized lateral size $t$ of the facets shown in Figure S5be. The ensemble of curves in the lower part belongs to the case where all edge forming Pd atoms contribute to the adsorption of oxygen. The ensemble in the upper part belongs to the case where each second $\mathrm{Pd}$ atom contributes to the adsorption.

We clearly stress here that even half of the edge forming Pd atoms is already a quite high atom number: we believe that the graphene should partially cover also the edges of the facets such that the actual number of active $\mathrm{Pd}$ atoms is lower at the edge. On the other side, we observe some nanometer long linear graphene defects on the top of the facets (see main article and Ref. [12]) where a large number of active Pd sites can be possibly found. Nevertheless, the simple estimation here shall merely demonstrate that the ratio of pressures $\frac{p_{\mathrm{O} 2, \mathrm{PdNP}}}{p_{\mathrm{O} 2, \mathrm{G} @ \mathrm{PdNP}}} \approx 100$ can be explained in principle by the number of limited active Pd sites, which are not covered by the graphene and which are needed to dissociate and adsorb oxygen. A more detailed $\mathrm{STM} / \mathrm{nc}-\mathrm{AFM}$ study is needed where the number of defects at the edges and on the facets can be counted with help of atomically resolved images.

Density Functional Theory Calculations. Figure S7 summarizes all atomic oxygen adsorption configurations on $\mathrm{Pd}(111)$ and $\mathrm{Pd}_{6} \mathrm{C}(111)$, which are discussed in the main article. The gray rhombus shows the $2 \sqrt{3} \times 2 \sqrt{3}$ unit cell used for the calculations. Figure S7a-d and Figure S7e-h show the four different oxygen coverages $(1 / 12,2 / 12,3 / 12$ and $4 / 12 \mathrm{ML})$ on $\operatorname{Pd}(111)$ and $\sqrt{3} \times \sqrt{3}$ graphene/ $\operatorname{Pd}(111)$, respectively. Figure S7i-l and Figure S7m-p show same coverages on the $\mathrm{Pd}_{6} \mathrm{C}(111)$ and $\sqrt{3} \times \sqrt{3}$ graphene $/ \mathrm{Pd}_{6} \mathrm{C}(111)$ surface, respectively.

Figure S8 shows the well known $0.25 \mathrm{ML} 2 \times 2$ oxygen coverage on $\mathrm{Pd}(111)$, which is frequently described in literature. The WF of this surface is within $0.005 \mathrm{eV}$ the same $\mathrm{WF}$, which is found for the $0.25 \mathrm{ML}$ oxygen coverage shown in Figure S7c. 


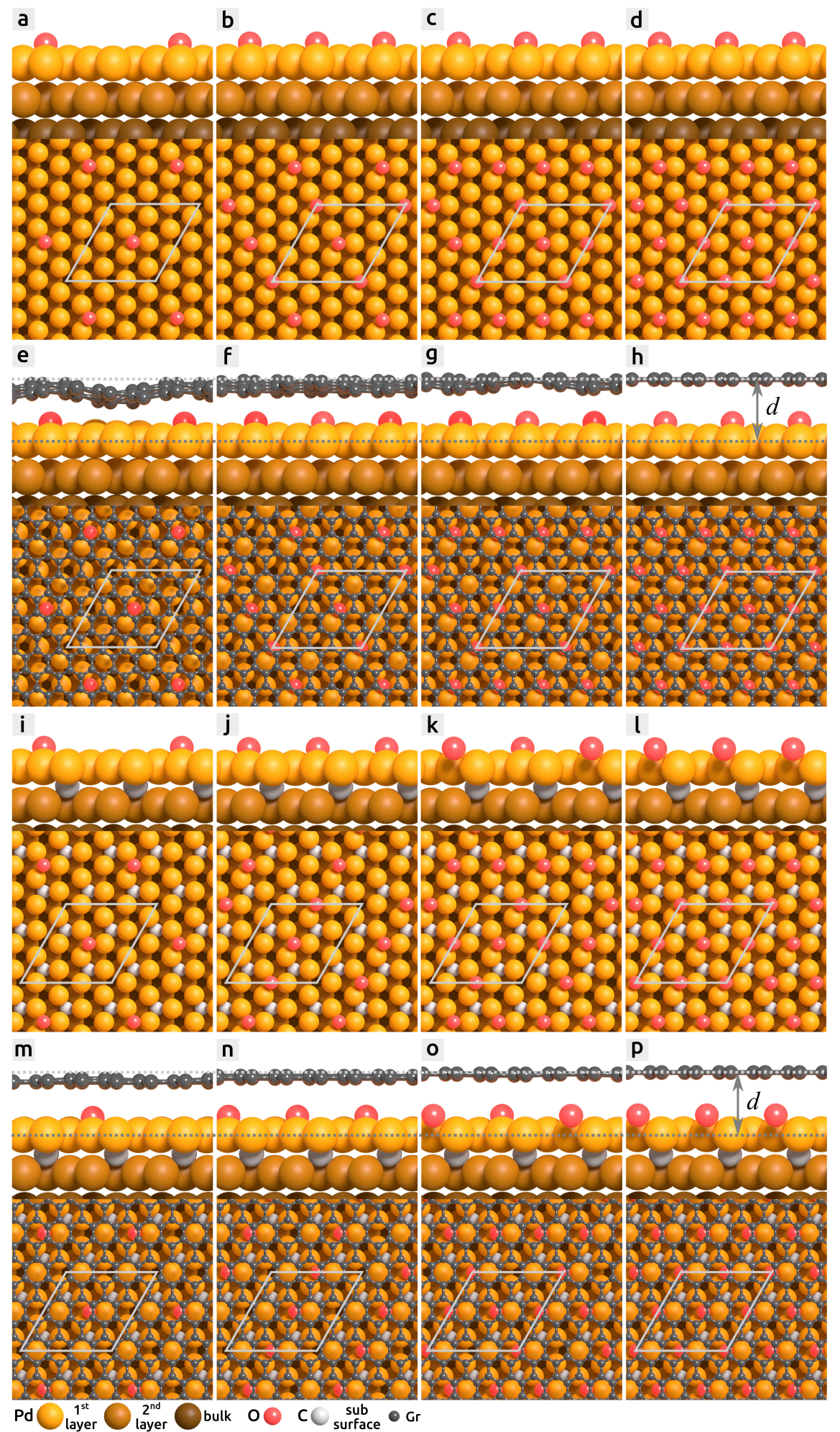

Figure S7: All details can be found in the text. 


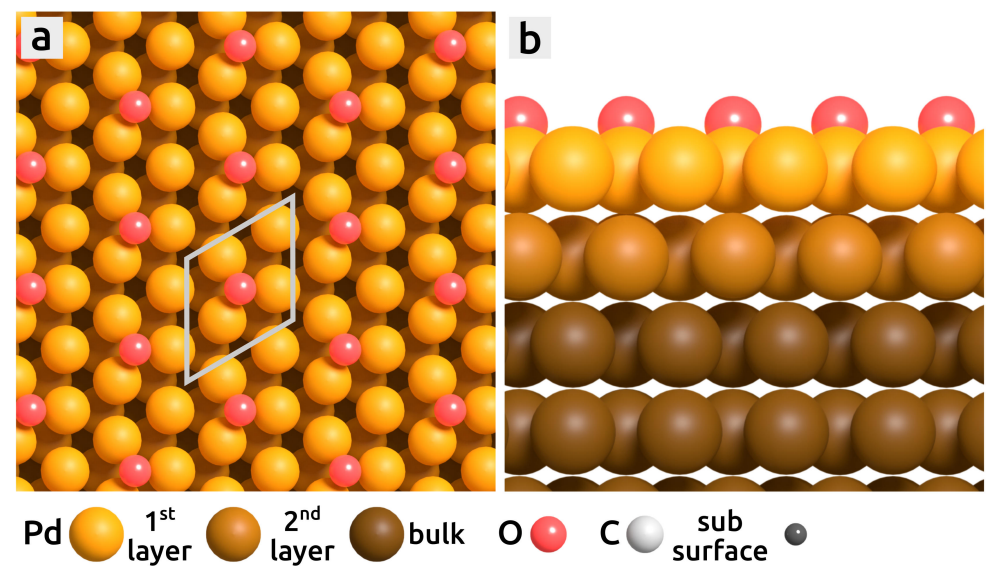

Figure S8: All details can be found in the text. 


\section{References}

1. Martinez-Martin, D.; Longuinhos, R.; Izquierdo, J. G.; Marele, A.; Alexandre, S. S.; Jaafar, M.; GómezRodríguez, J. M.; Bañares, L.; Soler, J. M.; Gomez-Herrero, J. Atmospheric Contaminants on Graphitic Surfaces. Carbon 2013, 61, 33-39.

2. Barth, C.; Claeys, C.; Henry, C. R. Surface Preparation of Hard Ionic Crystals by Ultrahigh Vacuum Cleavage. Rev. Sci. Instr. 2005, 76, 083907.

3. Lehmann, J.; Merschdorf, M.; Thon, A.; Voll, S.; Pfeiffer, W. Properties and Dynamics of the Image Potential States on Graphite Investigated by Multiphoton Photoemission Spectroscopy. Phys. Rev. B 1999, 60, 17037-17045.

4. Grönbeck, H.; Barth, C. Revealing Carbon Phenomena at Palladium Nanoparticles by Analyzing the Work Function. J. Phys. Chem. C 2019, 123, 4360-4370.

5. Lubuntu, Canonical (London, UK) (2019).

6. Barth, C. X11-Forwarding: How to Change Strange Colors? Asked on stackoverflow.com (message $35574154), 2016$.

7. The Gwyddion Open Source Project, 2019; http://gwyddion.net/.

8. The Matplotlib Open Source Project, 2019; http://www.matplotlib.org/.

9. The Python Open Source Project, 2019; https://www.python.org/.

10. Kitamura, S.; Suzuki, K.; Iwatsuki, M. High Resolution Imaging of Contact Potential Difference Using a Novel Ultrahigh Vacuum Non-Contact Atomic Force Microscope Technique. Appl. Surf. Sci. 1999, 140, 265-270.

11. Palacios-Lidon, E.; Henry, C. R.; Barth, C. Kelvin Probe Force Microscopy in Surface Chemistry: Reactivity of Pd Nanoparticles on Highly Oriented Pirolytic Graphite. ACS Catal. 2014, 4, 1838-1844.

12. Barth, C. Carbon Precursor Structures and Graphene on Palladium Nanoparticles. J. Phys. Chem. C 2018, 122, 522-529.

13. Kwon, S.-Y.; Ciobanu, C. V.; Petrova, V.; Shenoy, V. B.; Bareno, J.; Gambin, V.; Petrov, I.; Kodambaka, S. Growth of Semiconducting Graphene on Palladium. Nano Lett. 2009, 9, 3985-3990.

14. Murata, Y.; Starodub, E.; Kappes, B. B.; Ciobanu, C. V.; Bartelt, N. C.; McCarty, K. F.; Kodambaka, S. Orientation-Dependent Work Function of Graphene on Pd(111). Appl. Phys. Lett. 2010, 97, 143114.

15. We have used DMol, version 4.0.

16. Delley, B. From Molecules to Solids With the DMol ${ }^{3}$ Approach. J. Chem. Phys. 2000, 113, 7756-7764.

17. Delley, B. Hardness Conserving Semilocal Pseudopotentials. Phys. Rev. B 2002, 66, 155125. 
18. Vosko, S. H.; Wilk, L.; Nusair, M. Accurate Spin-Dependent Electron Liquid Correlation Energies for Local Spin Density Calculations: A Critical Analysis. Can. J. Phys. 1980, 58, 1200.

19. Perdew, J. P.; Burke, K.; Ernzerhof, M. Generalized Gradient Approximation Made Simple. Phys. Rev. Lett. 1996, 77, 3865.

20. Singh-Miller, N. E.; Marzari, N. Surface Energies, Work Functions, and Surface Relaxations of Low-Index Metallic Surfaces From First Principles. Phys. Rev. B. 2009, 80, 235407.

21. Patra, A.; Bates, J. E.; Sun, J.; Perdew, J. P. Properties of Real Metallic Surfaces: Effects of Density Functional Semilocality and van der Waals Nonlocality. Proc. Natl. Acad. Sci. 2017, 114, E9188-E9196.

22. Neugebauer, J.; Scheffler, M. Adsorbate-Substrate and Adsorbate-Adsorbate Interactions of Na and K Adlayers on Al(111). Phys. Rev. B. 1992, 46, 16067.

23. Broyden, C. G. The Convergence of a Class of Double-Rank Minimization Algorithms: 2. The New Algorithm. IMA J. Appl. Math. 1970, 6, 222-231.

24. Fletcher, R. A New Approach to Variable Metric Algorithms. Comp. Journal 1970, 13, 317-322.

25. Goldfarb, D. A Family of Variable-Metric Methods Derived by Variational Means. Math. Comp. 1970, 24, $23-26$.

26. Shanno, D. F. Conditioning of Quasi-Newton Methods for Function Minimization. Math. Comp. 1970, 24, 647-656.

27. Sque, S. J.; Jones, R.; Briddon, P. R. The Transfer Doping of Graphite and Graphene. Phys. Status Solidi Appl. Mater. Sci. 2007, 204, 3078-3084.

28. Takahashi, T.; Tokailin, H.; Sagawa, T. Angle-Resolved Ultraviolet Photoelectron Spectroscopy of the Unoccupied Band Structure of Graphite. Phys. Rev. B 1985, 32, 8317-8324.

29. Claessen, R.; Carstensen, H.; Skibowski, M. Conduction-Band Structure of Graphite Single Crystals Studied by Angle-Resolved Inverse Photoemission and Target-Current Spectroscopy. Phys. Rev. B 1988, $38,12582-12588$.

30. Ago, H.; Kugler, T.; Cacialli, F.; Petritsch, K.; Friend, R. H.; Salaneck, W. R.; Ono, Y.; Yamabe, T.; Tanaka, K. Workfunction of Purified and Oxidised Carbon Nanotubes. Synth. Met. 1999, 103, 24942495.

31. Ago, H.; Kugler, T.; Cacialli, F.; Salaneck, W. R.; Shaffer, M. S. P.; Windle, A. H.; Friend, R. H. Work Functions and Surface Functional Groups of Multiwall Carbon Nanotubes. J. Phys. Chem. B 1999, 103, 8116-8121. 
32. Suzuki, S.; Bower, C.; Watanabe, Y.; Zhou, O. Work Functions and Valence Band States of Pristine and Cs-Intercalated Single-Walled Carbon Nanotube Bundles. Appl. Phys. Lett. 2000, 76, 4007-4009.

33. Moos, G.; Gahl, C.; Fasel, R.; Wolf, M.; Hertel, T. Anisotropy of Quasiparticle Lifetimes and the Role of Disorder in Graphite From Ultrafast Time-Resolved Photoemission Spectroscopy. Phys. Rev. Lett. 2001, 87, 267402.

34. Hashimoto, H.; Watanabe, M.; Nishiuma, S.; Nakamura, K.; Yoshida, S. High-Resolution AES Study of $\mathrm{Ar}^{+}$-Irradiated Graphite. Surf. Interface Anal. 2003, 35, 19-23.

35. Koguchi, Y.; Meguro, T.; Hida, A.; Takai, H.; Maeda, K.; Yamamoto, Y.; Aoyagi, Y. Modification of Highly Oriented Pyrolytic Graphite (HOPG) Surfaces With Highly Charged Ion (HCI) Irradiation. Nucl. Instruments Methods Phys. Res. Sect. B Beam Interact. with Mater. Atoms 2003, 206, 202-205.

36. Yamamoto, R.; Yamada, T.; Taguchi, M.; Miyakubo, K.; Kato, H. S.; Munakata, T. Dispersions of Image Potential States on Surfaces of Clean Graphite and Lead Phthalocyanine Film. Phys. Chem. Chem. Phys. 2012, 14, 9601.

37. Ooi, N.; Rairkar, A.; Adams, J. B. Density Functional Study of Graphite Bulk and Surface Properties. Carbon 2006, 44, 231-242.

38. Demuth, J. E. Ultraviolet Photoemission Studies of Hydrogen Chemisorption Bonding to Ni, Pd and Pt Surfaces. Surf. Sci. 1977, 65, 369-375.

39. Behm, R. J.; Christmann, K.; Ertl, G.; van Hove, M. A. Adsorption of CO on $\operatorname{Pd(100).~J.~Chem.~Phys.~}$ 1980, 73, 2984-2995.

40. Wandelt, K.; Hulse, J. E. Xenon Adsorption on Palladium. I. The Homogeneous (110), (100), and (111) Surfaces. J. Chem. Phys. 1984, 80, 1340-1351.

41. Sesselmann, W.; Woratschek, B.; Küppers, J.; Ertl, G.; Haberland, H. Interaction of Metastable NobleGas Atoms With Transition-Metal Surfaces: Resonance Ionization and Auger Neutralization. Phys. Rev. $B$ 1987, 35, 1547-1559.

42. Kubiak, G. D. Two-Photon Photoelectron Spectroscopy of $\mathrm{Pd}(111)$. J. Vac. Sci. Technol. A 1987, 5, $731-734$.

43. Fischer, R.; Schuppler, S.; Fischer, N.; Fauster, T.; Steinmann, W. Image States and Local Work Function for $\mathrm{Ag} / \mathrm{Pd}(111)$. Phys. Rev. Lett. 1993, 70, 654-657.

44. Schäfer, A.; Shumay, I. L.; Wiets, M.; Weinelt, M.; Fauster, T.; Chulkov, E. V.; Silkin, V. M.; Echenique, P. M. Lifetimes of Unoccupied Surface States on $\mathrm{Pd}(111)$. Phys. Rev. B 2000, 61, 1315913163. 
45. Methfessel, M.; Hennig, D.; Scheffler, M. Trends of the Surface Relaxations, Surface Energies, and Work Functions of the 4 d Transition Metals. Phys. Rev. B 1992, 46, 4816-4829.

46. Skriver, H. L.; Rosengaard, N. M. Surface Energy and Work Function of Elemental Metals. Phys. Rev. $B$ 1992, 46, 7157-7168.

47. Heinrichsmeier, M.; Fleszar, A.; Hanke, W.; Eguiluz, A. G. Nonlocal Density-Functional Calculations of the Surface Electronic Structure of Metals: Application to Aluminum and Palladium. Phys. Rev. B 1998, 57, 14974-14982.

48. Pallassana, V.; Neurock, M.; Hansen, L. B.; Hammer, B.; Nørskov, J. K. Theoretical Analysis of Hydrogen Chemisorption on $\operatorname{Pd}(111), \operatorname{Re}(0001)$ and $\mathrm{Pd}_{\mathrm{ML}} / \operatorname{Re}(0001), \operatorname{Re}_{\mathrm{ML}} / \mathrm{Pd}(111)$ Pseudomorphic Overlayers. Phys. Rev. B 1999, 60, 6146-6154.

49. Ishida, H. Surface-Embedded Green-Function Method: A Formulation Using a Linearized-AugmentedPlane-Wave Basis Set. Phys. Rev. B 2001, 63, 165409.

50. Merrick, I.; Inglesfield, J. E.; Attard, G. A. Local Work Function and Induced Screening Effects at Stepped Pd Surfaces. Phys. Rev. B 2005, 71, 085407.

51. Da Silva, J. L. F.; Stampfl, C.; Scheffler, M. Converged Properties of Clean Metal Surfaces by AllElectron First-Principles Calculations. Surf. Sci. 2006, 600, 703-715.

52. Giovannetti, G.; Khomyakov, P. A.; Brocks, G.; Karpan, V. M.; van den Brink, J.; Kelly, P. J. Doping Graphene With Metal Contacts. Phys. Rev. Lett. 2008, 101, 026803.

53. Khomyakov, P. A.; Giovannetti, G.; Rusu, P. C.; Brocks, G.; van den Brink, J.; Kelly, P. J. FirstPrinciples Study of the Interaction and Charge Transfer Between Graphene and Metals. Phys. Rev. B 2009, 79 .

54. Hull, A. W. X-Ray Crystal Analysis of Thirteen Common Metals. Phys. Rev. 1921, 17, 571-588.

55. McKeehan, L. W. The Crystal Structures of the System Palladium-Hydrogen. Phys. Rev. 1923, 21, $334-342$.

56. Davey, W. P. Precision Measurements of the Lattice Constants of Twelve Common Metals. Phys. Rev. 1925, 25, 753 .

57. Barth, T.; Lunde, G. Die Gitterkonstanten der Platinmetalle, Silber und Gold. 1926, 121, 78-102.

58. Bredig, G.; Allolio, R. Röntgenuntersuchungen an Katalytisch Wirkenden Metallen. 1927, 126, 41-71.

59. Owen, E. A.; Yates, E. L. Precision Measurements of Crystal Parameters. The London, Edinburgh, and Dublin Philosophical Magazine and Journal of Science 1933, 15, 472-488. 
60. Maeland, A.; Flanagan, T. B. Lattice Constants and Thermodynamic Parameters of the HydrogenPlatinum-Palladium and Deuterium-Platinum-Palladium Systems. J. Phys. Chem. 1964, 68, 1419-1426.

61. Maeland, A.; Flanagan, T. B. The Hydrogen-Palladium System. Platinum Metals Rev. 1966, 10, 20-24.

62. Ellner, M. Zusammenhang zwischen Strukturellen und Thermo-Dynamischen Eigenschaften bei Phasen der Kupfer-Familie in $\mathrm{T}^{10}-\mathrm{B}^{4}$-Systemen. J. Less Common Met. 1981, 78, 21-32.

63. Mukhopadhyay, R.; Dasannacharya, B. A.; Nandan, D.; Singh, A. J.; Iyer, R. M. Real Time Deuterium Loading Investigation in Palladium Using Neutron Diffraction. Solid State Commun. 1990, 75, 359-362.

64. Akiba, H.; Kofu, M.; Kobayashi, H.; Kitagawa, H.; Ikeda, K.; Otomo, T.; Yamamuro, O. Nanometer-Size Effect on Hydrogen Sites in Palladium Lattice. J. Am. Chem. Soc. 2016, 138, 10238-10243.

65. Eichler, A.; Mittendorfer, F.; Hafner, J. Precursor-Mediated Adsorption of Oxygen on the (111) Surfaces of Platinum-Group Metals. Phys. Rev. B 2000, 62, 4744-4755.

66. Stewart, D. A. Ab Initio Investigation of Phonon Dispersion and Anomalies in Palladium. New J. Phys. 2008, 10, 043025 .

67. Haas, P.; Tran, F.; Blaha, P. Calculation of the Lattice Constant of Solids With Semilocal Functionals. Phys. Rev. B 2009, 79, 085104.

68. Csonka, G. I.; Perdew, J. P.; Ruzsinszky, A.; Philipsen, P. H. T.; Lebègue, S.; Paier, J.; Vydrov, O. A.; Ángyán, J. G. Assessing the Performance of Recent Density Functionals for Bulk Solids. Phys. Rev. B 2009, 79, 155107.

69. Harl, J.; Schimka, L.; Kresse, G. Assessing the Quality of the Random Phase Approximation for Lattice Constants and Atomization Energies of Solids. Phys. Rev. B 2010, 81, 115126.

70. Clay, J. P.; Greeley, J. P.; Ribeiro, F. H.; Nicholas Delgass, W.; Schneider, W. F. DFT Comparison of Intrinsic WGS Kinetics Over Pd and Pt. J. Catal. 2014, 320, 106-117.

71. Michaelson, H. B. The Work Function of the Elements and Its Periodicity. J. Appl. Phys. 1977, 48, 4729-4733.

72. Dillon, J. A.; Farnsworth, H. E. Work Function and Sorption Properties of Silicon Crystals. J. Appl. Phys. 1958, 29, 1195-1202.

73. Sgiarovello, C.; Binggeli, N.; Baldereschi, A. Influence of Surface Morphology on the Si(100) and (111) Ionization Potentials. Phys. Rev. B 2001, 64, 195305.

74. Allen, F. G.; Gobeli, G. W. Work Function, Photoelectric Threshold, and Surface States of Atomically Clean Silicon. Phys. Rev. 1962, 127, 150-158. 
75. Petermann, U.; Baikie, I. D.; Lägel, B. Kelvin Probe Study of Metastable States During Initial Oxygen Adsorption Dynamics on Si(111) 7×7. Thin Solid Films 1999, 343-344, 492-494.

76. Baikie, I.; Petermann, U.; Lägel, B. UHV-Compatible Spectroscopic Scanning Kelvin Probe for Surface Analysis. Surf. Sci. 1999, 433-435, 249-253.

77. Raisin, C.; Vieujot-Testemale, E.; Brahim, A. B.; Palau, J. M.; Lassabatere, L. Work Function Measurements During Growth of Ultra Thin Films of $\mathrm{SiO}_{2}$ on Characterized Silicon Surfaces. Solid. State. Electron. 1984, 27, 413-417.

78. Derry, G. N.; Kern, M. E.; Worth, E. H. Recommended Values of Clean Metal Surface Work Functions. J. Vac. Sci. Technol. A 2015, 33, 060801.

79. He, J.-W.; Norton, P. Thermal Desorption of Oxygen From a Pd(110) Surface. Surf. Sci. 1988, 204, $26-34$.

80. Ulbricht, H.; Zacharia, R.; Cindir, N.; Hertel, T. Thermal Desorption of Gases and Solvents From Graphite and Carbon Nanotube Surfaces. Carbon 2006, 44, 2931-2942. 